\title{
Translation Correctness for First-Order Object-Oriented Pattern Matching
}

\author{
Burak Emir ${ }^{1}$, Qin $\mathrm{Ma}^{2}$, and Martin Odersky ${ }^{1}$ \\ ${ }^{1}$ EPFL, 1015 Lausanne, Switzerland \\ 2 OFFIS, Escherweg 2, Oldenburg, Germany \\ LAMP-REPORT-2007-003
}

\begin{abstract}
Pattern matching makes ML programs more concise and readable, and these qualities are also sought in object-oriented settings. However, objects and classes come with open class hierarchies, extensibility requirements and the need for data abstraction, which all conflict with matching on concrete data types. Extractor-based pattern matching has been proposed to address this conflict. Extractors are user-defined methods that perform the task of value discrimination and deconstruction during pattern matching. In this paper, we give the first formalization of extractor-based matching, using a first-order object-oriented calculus. We give a direct operational semantics and prove it sound. We then present an optimizing translation to a target language without matching, and prove a correctness result stating that an expression is equivalent to its translation.
\end{abstract}

\section{Introduction}

Algebraic datatypes and pattern matching render ML programs more concise, easier to read, and amenable to mathematical proof by structural induction [1]. Match expressions are highlevel constructs with good properties: Compilers can translate them very efficiently [2-6]. However, ML style pattern matching is often incompatible with data abstraction.

To see why, consider the ML datatype definition in Fig. 1. which introduces a type name and its constructors. Constructors play the double role of tags and functions that aggregate data. Every list instance is tagged with either Nil or Cons. In match expressions, values are distinguished by their tag to recover their data. In the example, the append function concatenates lists by matching its argument xs: if it is the empty list, we return the second argument zs. If xs is a Cons cell, a non-destructive update appends zs to its tail via a recursive call.

This style of programming is concise and readable. The programs can be efficient, since tags can be translated to integers and match expressions to cascaded switch statements. However, note that the set of constructors as well as the arrangement of data items is fixed once and for all. Furthermore, the grouping of data items for the Cons constructor (e.g. int $*$ list) exposes the representation of the data, making it harder to change.

Representation independence forms the basis for data abstraction. Researchers have suggested to reconcile pattern matching and data abstraction [7, 8]. However, slow adoption suggests that data abstraction, while desirable, is not considered essential for functional programming. In object-oriented programming, data abstraction is indispensable: it is the one of the 


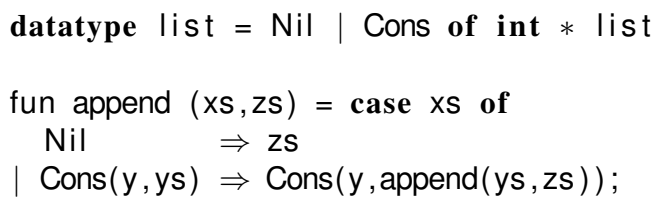

Fig. 1. Algebraic Matching in ML

key mechanisms that permit object-oriented programmers to describe complex systems using class hierarchies.

Pattern matching in object-oriented systems is appropriate when the set of operations cannot be anticipated, as witnessed by the Visitor pattern [9]. Like functional pattern matching, the Visitor pattern breaks encapsulation: the internal representation of objects has to be exposed in order for operations like append to access them.

In order to use pattern matching without tying it to a closed set of types that expose their representation, a semantics for pattern matching based on user-defined functions has been proposed independently by Emir, Odersky and Williams [10], and Syme, Neverov and Margetson [11]. A pattern is interpreted as invocation of a so-called extractor method, which discriminates and deconstructs the input value. This decouples the type of an accepted value from the representation extracted from it.

A toy example of matching with extractors is given in Fig. 2. It defines a List class, three subclasses Nil, Cons, and Singleton as well as two extractors cons and nil. These extractors are methods, which here use test expressions $a ?\{\mathrm{x}: C \Rightarrow d\} /\{e\}$. Such a test expression is the contraction of the Java code

$$
\text { if ( } a \text { instanceof } C)\{C \times=(C) a ; d\} \text { else } e \text {. }
$$

The append implementation demonstrates the use of extractors. A pattern invokes an extractor methods with an implicit argument — the xs.cons $(y, y s)$ pattern expresses two steps:

1. invoke method $x$ s.cons with the input value $x s$ of the match expression as single argument

2. if it returns null, the input value is rejected. Otherwise, it is accepted: bind the first field of the result to $y$ and the second one to ys.

The return value of the extractor is a representation object, used for matching subpatterns or binding the constituents to local variables. Here, the matched type Cons is used in internal and external representation of lists. Representation can be chosen independently though, only a suitable extractor implementation needs to be provided. Here, lists that contain only one element can internally be represented with class Singleton, which is never exposed through pattern matching. This class could have been added later, without breaking client code that references cons and nil extractors.

We were curious whether algorithms for optimized translation of pattern matching can be applied to extractors to work in an object-oriented context. Also, the question arises which conditions (if any) have to be satisfied by extractors in order to prove that translation preserves the meaning of the program. 


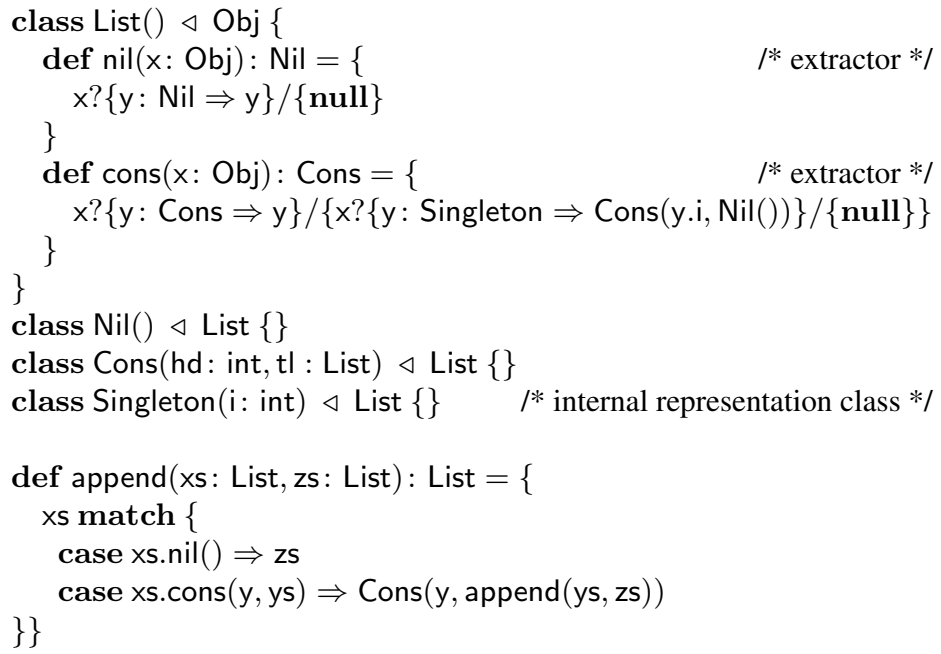

Fig. 2. Extractor-based Matching in the FPat Calculus

In this paper, we present possible answers to these questions. We adapted the translation to decision-trees following Pettersson [4]. Using a formal calculus based on Featherweight Java (FJ) [12], we define a first-order object-oriented language that offers runtime type inspection and pattern matching. We give a direct operational semantics and then show a straightforward translation algorithm to compile match expressions down to the fragment without pattern matching. This translation is proven correct, under the hypothesis that extractor methods do not diverge and do not throw exceptions. To the best of our knowledge, this hypothesis was never mentioned in the literature, though its necessity is easily justified in our development.

Extractor-based pattern matching has been implemented independently in $\mathrm{F}^{\sharp}$ (there called "active recognizer") and Scala. In contrast to previous work, this paper aims to shed light on its formal underpinnings. In summary, we contribute:

- a formal calculus that precisely describes extractor-based pattern matching,

- a formal definition and correctness proof of an optimized translation of match expressions,

- and formal conditions extractors have to satisfy in order for optimization to be correct.

The rest of the paper is organized as follows. We define FPat and in Section 2. We describe the translation of pattern matching in Section 3. We describe the correctness proof in Section 4. Section 5 discusses related work and Section 6 concludes.

\section{An Object-Oriented Calculus with Pattern Matching}

We define FPat by describing its syntax, typing rules and operational semantics. The syntax and semantics are given in Fig. 3, and the typing rules and auxiliary definitions are given in Fig. 4 and Fig. 5. The calculus is based on FJ, but with semantics defined using a strict, left-to-right big-step semantics. We then define divergent programs and show type soundness. 


\subsection{Syntax}

What follows is a short presentation of the rules and notation. A sequence $\alpha_{1} . . \alpha_{n}$ is abbreviated as $\alpha_{\star}^{1 . . n}$, where $\alpha$ can be an expression, a name-type binding, or a judgment. The empty sequence is written $\bullet$. Multiple occurence of the $\star$ indicate that the same index appears at multiple positions. Moreover, we shall need to express sequences with holes, so $\alpha_{\star}, \beta, \alpha_{\star}{ }^{1 . . .] i[. n}$ stands for $\alpha_{\star}^{1 . . i-1}, \beta, \alpha_{\star}^{i+1 . . n}$.

An FPat program $c d_{\star}^{1 . . n} ; e$ is a set of class definitions and a top-level expression. Class definitions are kept in a class table, which we leave implicit throughout the paper and which satisifies the important property of absence of inheritance cycles and duplicate entries. Classes have an explicit superclass as well as field declarations and method definitions, all publicly accessible. Methods can have a @safe annotation to indicate that they terminate without throwing an exception. The class hierarchy induces a subtype relation $<$ :, of which the magic class Obj forms the largest element and the magic class Exc forms the smallest. These two types are magic because they do not have definitions in the class table. We also have a least upper bound $C \sqcup D$ operation, which is the least type $E$ in the hierarchy that satisfies $C<: E$ and $D<: E$.

There are 8 expression forms: null, variables $x$, field selection e.f, method invocation e.m $\left(e_{\star}^{1 . . n}\right)$, object construction $C\left(e_{\star}^{1 . . n}\right)$, exception throw, test expressions $a ?\{\mathrm{x}: C \Rightarrow$ $d\} /\{e\}$ and match expressions $e_{\star}^{1 . . n}$ match $\left\{c_{\star}^{1 . . k}\right\}$. The calculus does not model assignment nor object identity. The free variables $f v$ and the defined variables $d v$ are defined in the straightforward manner.

\subsection{Semantics}

We briefly describe operational semantics of the fragment without pattern matching, in order to be self-contained. Semantics specific to pattern matching are deferred to a separate section below.

Terminating computation of meaningful expressions is modeled by a big-step evaluation relation $e \Downarrow q$ that takes expressions $e$ to results $q$. A result $q$ is either values $v \in$ Values, the null result or the exception throw. Note that substitutions are all restricted to map variables only to values or null.

A value is the outcome of an object construction $C\left(v_{\star}^{1 . . n}\right)$, which is written without new. There is no explicitly declared constructor, instead the field order determined by the inheritance hierarchy (specified in the auxiliary judgment fields $(C)$ ) is used. The following correct program may serve as an example as to how object construction relates to class definitions:

$$
\begin{aligned}
& \text { class } D(\mathrm{f}: A) \triangleleft \mathrm{Obj}\{\ldots\} \\
& \text { class } C(\mathrm{~g}: B) \triangleleft D\{\ldots\} \\
& C(A(), B())
\end{aligned}
$$

Rules (Rfld), (Rinvk), (Rnew) describe field access, method invocation and object construction. The auxiliary judgment mbody $(\mathrm{m}, C)$ specifies how to lookup method bodies. Rules (Cfld), (Crcv), (Carg), (Cnew) throw or propagate exceptions.

The only significant use of null happens in test expressions. Their behavior is specified in rules (Rcst) and (Rskp): if the tested expression, or scrutinee, is not null and its type lesser than the required type, it is bound to a local variable and the first branch is evaluated (Rcst). 


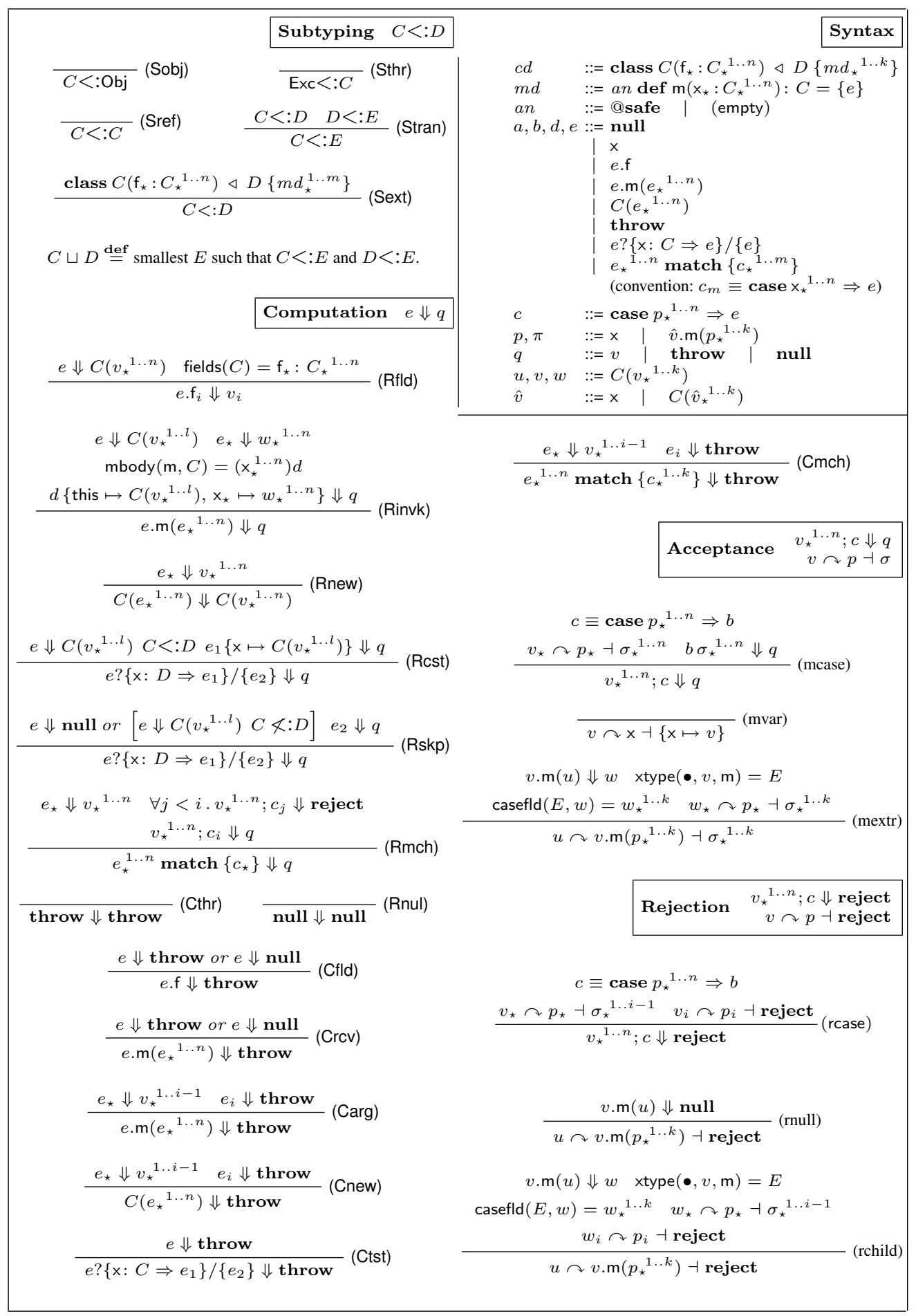

Fig. 3. FPat Syntax and Semantics 
Otherwise, the second branch is evaluated (Rskp). If the scrutinee throws, the exception is propagated (Ctst).

The relation $\Downarrow$ does not specify behavior of meaningless or non-terminating programs. To show type soundness, divergent programs are defined using a relation $\Uparrow$ in Fig. 6. Meaningless expressions are then precisely those that are neither terminating nor divergent. This is described in detail, after elaborating on pattern matching.

\subsection{Semantics of Matching}

Pattern matching expressions contain a number of (at least one) case clauses, each of which compares the $n$ input values against $n$ patterns, and the last clause may only have variable patterns. This excludes pathological expressions of the form $e_{\star}^{1 . . k}$ match \{\} and ensures that the behavior is defined for any possible combination of input values. This convention is easily enforced by a compiler.

Matching depends on judgments describing acceptance and rejection of patterns and cases. Rule (Rmch) describes evaluation of cases according to the first match policy: an accepting case is evaluated only if all preceding cases rejected the input.

Two separate judgments describe acceptance for cases $v_{\star}^{1 . . n} ; c \Downarrow q$ and patterns $v \curvearrowright p \dashv \sigma$. We explain the judgments for case clauses first. A case accepts and evaluates to result $q$ if each input value is accepted by the corresponding pattern (mcase). Analogousy, a case rejects $v_{\star}^{1 . . n} ; c \Downarrow$ reject if an initial segment of patterns accept and the following pattern rejects its input (rcase). Together, these rules describe a left-to-right evaluation of patterns. If a pattern accepts, it yields a substitution, and if all patterns accept, the combined substitution is applied to the body of the case (the merging of substitutions is indicated by juxtaposition).

The judgment $v \curvearrowright p \dashv \sigma$ describes that pattern $p$ accepts $v$ and yields substitution $\sigma$. Analogously, the judgment $v \curvearrowright p \dashv$ reject describes rejection. A variable pattern always accepts its input (mvar), yielding the obvious substitution. An extractor pattern accepts (mextr) if:

1. evaluation of the extractor call returns a value $w$, yielding so-called case fields $w_{\star}^{1}{ }^{1 . . k}$

2. all subpatterns accept the case fields, yielding substitutions $\sigma_{\star}^{1 . . k}$

The extractor pattern rejects if the call returns null (rnull) or if one of its subpattern rejects its input (rchild). Case fields casefld $(E, w)$ are determined for the return type $E$ of the extractor method, as specified in the auxiliary judgment $x \operatorname{type}(\bullet, v, \mathrm{~m})$. They are the fields declared in the class definition of $E$ itself, which thus serves as the "representation type".

The outcome is undefined when extractors throw exceptions. For this reason, the @safe modifier is required on any method that is referenced as an extractor. Safety is of course undecidable, but restrictions and approximations are available to tackle this problem. Our focus in this paper is on justifying the condition, not checking it.

Discussion In any statically-typed definition of pattern matching, order and types of subpatterns must be specified. The benefit of using extractors lies in decoupling the matched type from the representation type.

Pattern matching usually includes matching on literals like 42 , true and named constants like foo. While literal expressions are constructors without arguments, a corresponding convention for extractors can be assumed. Named constants are added by allowing tests for singleton types $v$.type. Structural equality then ensures that $C\left(w_{\star}^{1 . . k}\right) \in v$.type if $v \equiv C\left(w_{\star}^{1 . . k}\right)$. 


\subsection{Typing}

The type system is specified through a set of syntax-directed typing rules. The rules specific to matching are described in a separate section below.

Type judgments for expressions have the form $\Gamma \vdash e \in C$ where $\Gamma$ is a type environment (a finite mapping from variables to types), $e$ an expression and $C$ a class. The judgments $c d \diamond$ and an $m d \diamond$ in $C$ assert well-typedness of class and method definitions. Methods annotated with @safe are assumed to terminate and never throw exceptions for any input (including null). A class definition is well-typed if all its methods are well-typed, and a method is well-typed if its return expressions is well-typed under the appropriate type environment. If the method overrides a method in a superclass, their signatures have to be identical, which is asserted by the judgment override $\left(a n\left(B_{\star}^{1 . . n}\right) B, \mathrm{~m}, D\right)$. A program is well-typed if all its class definitions are well-typed, and its top-level expression is well-typed in the empty environment.

Typing expressions is straightforward. Rules (Tthr) and (Tnul) give the most specific type Exc to the throw and null results. (Tvar) takes the type of a variable from the type environment, and field access (Tfld) and object construction (Tnew) is checked against the fields of the class as calculated by the judgment fields $(C)$. A similar judgment for method signatures $\operatorname{mtype}(\mathrm{m}, C)$ is used to type-check method invocation (Tinvk). Thus, well-typed method calls and objects constructions have the right number and types of arguments.

Test expressions are checked with rule (Ttst), which modifies the type environment for the succeeding branch to account for the new local variable. Binding in test expressions can be used to define a derived form val $\mathrm{x}: C=a ; b$, which we will introduce later.

\subsection{Typing of Match Expressions}

Match expressions are well-typed if all their clauses are well-typed (Tmch), using the least upper bound to combine the clauses' result types. To type-check a single clause case $p_{\star}^{1 . . n} \Rightarrow b$ (Tcase), each pattern in $p_{\star}^{1 . . n}$ is type-checked w.r.t. the type environment $\Gamma$ and an "expected type" $C_{\star}$, yielding a type environment $\Delta_{\star}$ as in $\Gamma ; C_{\star} \ni p_{\star} \dashv \Delta_{\star}^{1 . . n}$. Then, the body is type-checked against the combined type environments as in $\Gamma, \Delta_{\star}^{1 \ldots n} \vdash b \in D$. Variables introduced in patterns must be pair-wise different and may not clash with $\Gamma$, which is implicit in the juxtaposition of environments.

Pattern typing $\Gamma ; E \ni p \dashv \Delta$ is type-checked as follows: For variable patterns (TPvar), the expected type $E$ is used to produce a singleton environment. For extractor patterns (TPextr), the judgment $\mathrm{xtype}(\Gamma, \hat{v}, \mathrm{~m})$ looks up the type of receiver and the signature of the extractor method in order to recover the representation type. It also ensures that extractors are @safe. The casefield types are then used as expected types to check the subpatterns. Then we merge the environments obtained from checking the subpatterns.

\subsection{Divergent Programs}

Our approach to type soundness, following Leroy and Grall [13], is to characterize meaningless programs as those that neither terminate nor diverge. Divergent programs are defined coinductively by the set of divergence rules in Fig. 6 . These rules are tailored to establish that any well-typed term that does not terminate necessarily diverges. Their coinductive nature is indicated by horizontal double lines: coinductive derivations are infinite trees with the root being the assertion to derive and the successors of each node being determined by a derivation rule. 


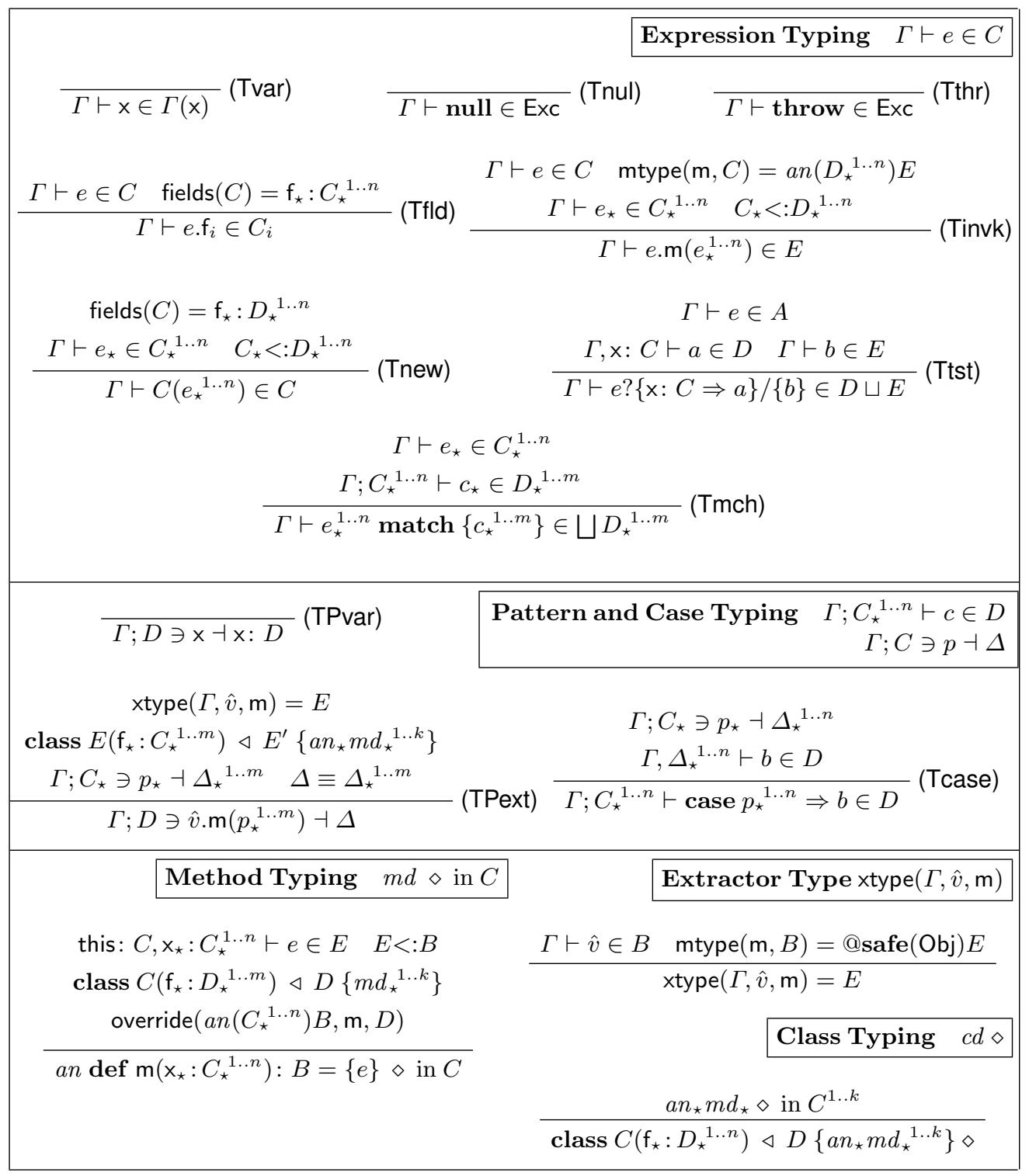

Fig. 4. Typing Rules 


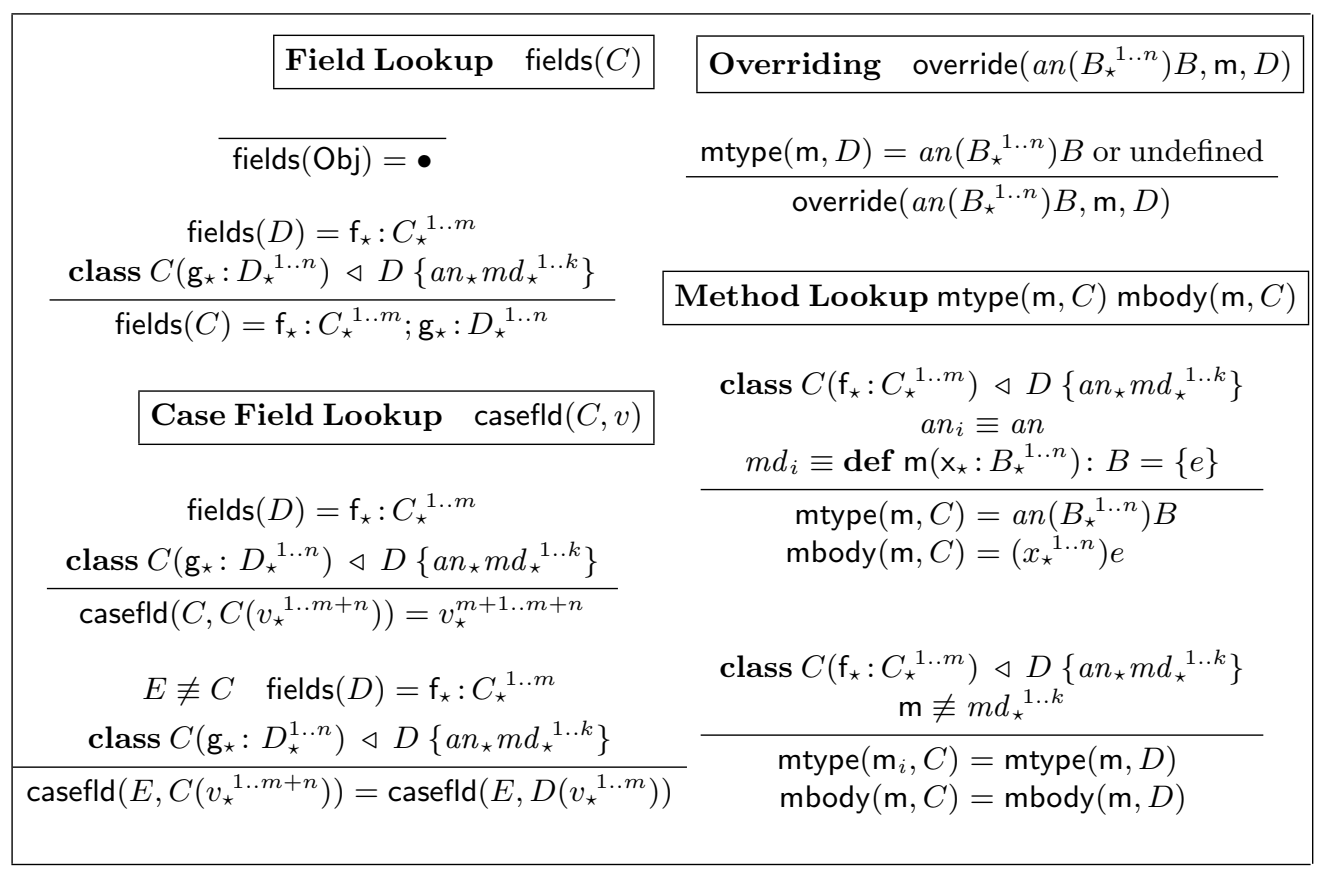

Fig. 5. Auxiliary Definitions

Let us consider the rules one by one. Rule (Dfld) and (Drcv) express that accessing a field or invoking a method on a divergent expression yields a divergent expression. Rules (Darg) and (Dnew) say that object construction and method invocation diverges if one of their arguments diverge. Note that a strict call-by-value, left-to-right evaluation order is followed also here. Rule (Dinvk) says that calling a method with arguments that make the method body diverge yields a divergent expression. Similarly, (Dtst), (Dcst) and (Dskp) characterize divergent test expressions by locating divergence in the respective subexpression.

In match expressions, a divergent match expression can be traced back to some (possibly empty) initial segment of rejecting case clauses followed by a divergent case clause. A case clause may only diverge because its body diverges (Dbdy).

Discussion It is possible but would be tedious if we omit the @safe hypothesis, give divergence rules for divergent extractor calls, and adapt the soundness proof accordingly.

If we did not rely on the @safe annotation, divergence could also be caused by extractor calls during pattern match evaluation. It is possible but tedious to omit the @ safe hypothesis, give divergence rules for divergent extractor calls and adapt the soundness proof accordingly. Moreover, we discover that @safe is actually needed for correct translation (see section 3.2). As a consequence, we chose the simpler route by defining only those divergent programs that are needed for Progress. 


$$
\begin{aligned}
& \text { Divergent Computation } e \Uparrow
\end{aligned}
$$

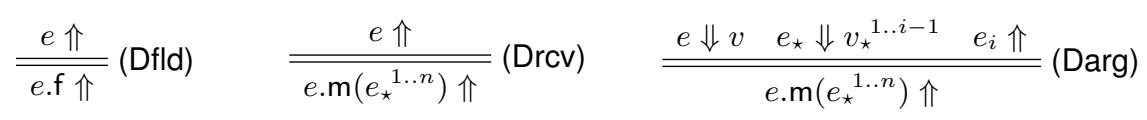

$$
\begin{aligned}
& e \Downarrow C\left(v_{\star}^{1 . . m}\right) \quad e_{\star} \Downarrow w_{\star}^{1 . . n} \\
& \operatorname{mbody}(C, \mathrm{~m})=\left(\mathrm{x}_{\star}^{1 . . n}\right) b \\
& b\left\{\text { this } \mapsto C\left(v_{\star}^{1 . . m}\right), x_{\star} \mapsto w_{\star}^{1 . . n}\right\} \Uparrow \\
& e . \mathrm{m}\left(e_{\star}^{1 . . n}\right) \Uparrow \\
& \frac{e \Uparrow}{e ?\{\mathrm{x}: C \Rightarrow a\} /\{b\} \Uparrow} \text { (Dtst) } \frac{e \Downarrow D\left(v_{\star}^{1 . . n}\right) \quad D<: C \quad a\left\{\mathrm{x} \mapsto D\left(v_{\star}^{1 . . n}\right)\right\} \Uparrow}{e ?\{\mathrm{x}: C \Rightarrow a\} /\{b\} \Uparrow} \text { (Dcst) } \\
& \frac{e \Downarrow \text { null or }\left[e \Downarrow D\left(v_{\star}^{1 . . n}\right) \quad D \nless: C\right] \quad b \Uparrow}{e ?\{\mathrm{x}: C \Rightarrow a\} /\{b\} \Uparrow} \text { (Dskp) } \\
& \frac{e_{\star} \Downarrow v_{\star}^{1 . . i-1} e_{i} \Uparrow}{e_{\star}^{1 . . n} \operatorname{match}\left\{c_{\star}^{1 . . m}\right\} \Uparrow}(\text { Dmch }) \\
& e_{\star} \Downarrow v_{\star}^{1 . . n} \quad \forall j<i . v_{\star}^{1 . . n} \curvearrowright c_{j} \dashv \text { reject } \\
& \frac{e_{\star} \Downarrow v_{\star}^{1 . . i-1} e_{i} \Uparrow}{C\left(e_{\star}^{1 . . n}\right) \Uparrow}(\text { Dnew }) \\
& \text { (Dinvk) } \\
& v_{\star}^{1 . . n} ; c_{i} \Uparrow e \\
& e_{\star}^{1 . . n} \operatorname{match}\left\{c_{\star}^{1 . . m}\right\} \Uparrow \\
& \text { Divergent Cases and Patterns } v_{\star}^{1 . . n} ; c \Uparrow e \\
& \frac{c=\text { case } p_{\star}^{1 . . n} \Rightarrow b \quad v_{\star} \curvearrowright p_{\star} \dashv \sigma_{\star}^{1 . . n} \quad b \sigma_{\star}^{1 . . n} \Uparrow}{v_{\star}^{1 . . n} ; c \Uparrow b \sigma_{\star}^{1 . . n}}(\mathrm{Dbdy})
\end{aligned}
$$

Fig. 6. Divergent Programs

\subsection{Soundness}

We now prove type soundness using big-step versions of the standard lemmata.

Lemma 1 (Uniqueness) For every $a$, if $a \Downarrow q$ then for all $q^{\prime}$, if $a \Downarrow q^{\prime}$ then $q=q^{\prime}$.

Lemma 2 (Termination) For all a and all $q$, it holds that if $a \Downarrow q$ then $a \nmid$ 仗

Lemma 3 (Subtypes have all Fields) If $C<: D, C \neq \operatorname{Exc}$ then fields $(C)=$ fields $(D) ; \mathrm{g}_{\star}: E_{\star}{ }_{\star} . . m$.

Lemma 4 (Subtypes have all Methods) If $C<: D, C \neq \operatorname{Exc}$ and $\operatorname{mtype}(\mathrm{m}, D)=\operatorname{an}\left(C_{\star}^{1 . . n}\right) B$, then mtype $(\mathrm{m}, C)=\operatorname{an}\left(C_{\star}^{1 . . n}\right) B$.

The following two lemmata are needed to prove the substitution lemma for pattern matching expressions. We have to deal with the typing rule for variables which might end up producing a "better" environment for input values whose type has become more precise after substitution. We write $\Delta^{\prime}<: \Delta$ when $\operatorname{dom}(\Delta)=\operatorname{dom}\left(\Delta^{\prime}\right)$ and $\mathrm{x}: B \in \Delta$ implies $\mathrm{x}: A \in \Delta^{\prime}$ with $A<: B$. 
Lemma 5 (Subtypes yield Refined Environment)

If $C<: D$ and $\Gamma ; D \ni p \dashv \Delta$ then $\Gamma ; C \ni p \dashv \Delta^{\prime}$ for some $\Delta^{\prime}<: \Delta$.

Lemma 6 (Refined Environment preserves Typing) If $C_{\star}<: D_{\star}^{1 . . n}$ and $\Gamma, \mathrm{x}_{\star}: D_{\star}^{1 . . n} \vdash e \in$ $B$ then $\Gamma, \mathrm{x}_{\star}: C_{\star}^{1 . . n} \vdash e \in A$ for $A<: B$.

Lemma 7 (Weakening) If $\Gamma \vdash d \in D$ and $\mathrm{x} \notin f v(d)$, then $\Gamma, \mathrm{x}: C \vdash d \in D$. for any $C$.

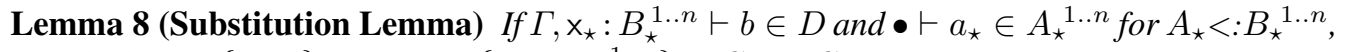
$a_{\star} \in$ Values $\cup\{$ null $\}$ then $\Gamma \vdash b\left\{\mathrm{x}_{\star} \mapsto a_{\star}^{1 . . n}\right\} \in C$, for $C<: D$.

Lemma 9 (Preservation)

If $a \Downarrow q$ and $\bullet \vdash a \in C$, then $\bullet \vdash q \in C^{\prime}$ for some $C^{\prime}<: C$.

The big-step version of the progress lemma uses coinduction.

Lemma 10 (Progress)

If $\bullet-a \in C$ and $a \Downarrow q$ for all $q$, then $a \Uparrow$.

Thm 1 (Type Soundness)

If $\bullet \vdash a \in C$ then either $a \Uparrow$ or $a \Downarrow q$ for some $q$ with $\bullet \vdash q \in C^{\prime}, C^{\prime}<: C$.

\section{Translation}

\subsection{Rewriting Match Expressions}

An elegant way to describe translation is to give a set of rewrite rules, which are applied successively until all match expressions are replaced with lower-level operations. Apart from being easy to understand and implement, correctness can then be established for each rule separately.

There are two approaches to the compilation of pattern matching, one based on decisiontrees and the other based on backtracking automata $[6,5]$. We chose the translation to decision trees, which in the functional setting guarantees that no input value is tested more than once. Our presentation of the algorithm follows Pettersson's [4].

The central idea is to remove a top-level pattern of a case clause, lifting its subpatterns to the top-level. Consider the two expressions below:

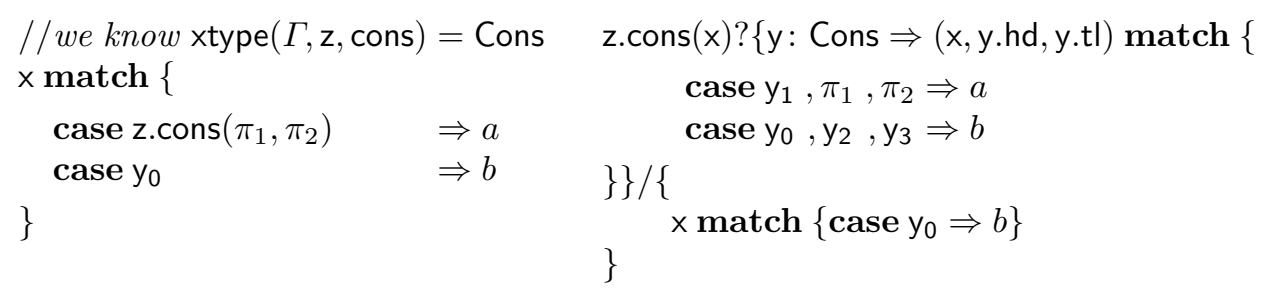

Some scrutiny reveals that these are actually equivalent. The extractor of the first pattern z.cons $\left(\pi_{1}, \pi_{2}\right)$ in the first case has been pulled out and a test is done on the outcome: if it is non-null, it is bound to the fresh variable $y$ and the subpatterns are matched against the case fields y.hd, y.tl. Note that the width of the original match is augmented by lifting the nested 


\section{Translation $\llbracket \Gamma \vdash a \operatorname{match}\{M\} \in D \rrbracket=e$}

(Tmp)

$\llbracket \frac{\ldots}{\Gamma \vdash a_{\star}^{1 . . n} \operatorname{match}\left\{c_{\star}^{1 . . m}\right\} \in D} \rrbracket=\operatorname{val} z_{\star}: C_{\star}=a_{\star}^{1 . . n} ; z_{\star}^{1 . . n} \operatorname{match}\left\{c_{\star}^{1 . . m}\right\}$

condition: $\quad-a_{\star}$ are not all variables

(Var)

$\llbracket \frac{\ldots \quad \Gamma \vdash \mathrm{z}_{\star} \in C_{\star}^{1 . . n}}{\Gamma \vdash \mathrm{z}_{\star}^{1 . . n} \operatorname{match}\left\{c_{\star}^{1 . . m}\right\} \in D} \rrbracket=b\left\{\mathrm{x}_{\star} \mapsto \mathrm{z}_{\star}^{1 . . n}\right\}$

condition: $\quad-c_{1}$ has the shape case $x_{\star}^{1 \ldots n} \Rightarrow b$

(Mix)

$\llbracket \frac{\ldots \quad \Gamma \vdash \mathrm{z}_{\star} \in C_{\star}^{1 . . n}}{\Gamma \vdash \mathrm{z}_{\star}^{1 . . n} \operatorname{match}\left\{c_{\star}^{1 . . m}\right\} \in D} \rrbracket=\hat{v} \cdot \mathrm{m}\left(\mathrm{z}_{i}\right) ?\left\{\mathrm{y}: C \Rightarrow \operatorname{val}_{\star} \mathrm{y}_{\star}: D_{\star}=\mathrm{y} \cdot \mathrm{f}_{\star}^{1 . . k} ; d\right\} /\{e\}$

condition: $\quad-c_{1}$ has the shape case $x_{\star}^{1 . . i-1} \hat{v} \cdot \mathrm{m}\left(p_{\star}^{11 . . k}\right) p_{\star}{ }^{i+1 . . n} \Rightarrow b$

translation steps:

- $\hat{v} \cdot \mathrm{m}\left(\mathrm{z}_{i}\right)$ has pattern typing $\frac{\mathrm{xtype}(\Gamma, \hat{v}, \mathrm{~m})=C \quad \ldots}{\Gamma ; C_{i} \ni \hat{v} \cdot \mathrm{m}\left(p_{\star}^{\prime \prime . k}\right) \dashv \Delta}$ (TPext)

- the definition of $C$ is class $C\left(\mathrm{f}_{\star}: D_{\star}^{1 . . k}\right) \triangleleft E\left\{m d_{\star}^{1 \ldots n}\right\}$

- $\mathrm{y}, \mathrm{y}_{\star}{ }^{1 . . k}$ are fresh variables

- $d=\mathrm{z}_{\star}, \mathrm{y}, \mathrm{y}_{\star}^{1 . . k}, \mathrm{z}_{\star}^{1 . .][i . . n} \operatorname{match}\left\{\operatorname{expand}_{\hat{v} . m}\left(c_{\star}^{1 \ldots . m}\right)\right\}$

- $e=\mathbf{z}_{\star}^{1 . . n}$ match $\left\{\right.$ other $\left._{\hat{v} . m}\left(c_{\star}^{1 . . m}\right)\right\}$

- where $\operatorname{expand}_{\hat{v} . m}$ and other $\hat{v} . m$ are defined for arbitrary patterns $p_{\star}, p_{\star}^{\prime}$, as (subscript omitted):

$\operatorname{expand}(\bullet)=\bullet$

$\operatorname{expand}\left(\right.$ case $\left.p_{\star} \hat{v} \cdot \mathrm{m}\left(p_{\star}^{1 \ldots . k}\right) p_{\star}^{1 \ldots .] i[. . n} \Rightarrow b ; c_{\star}^{1 \ldots . m}\right)=$

case $p_{\star} \mathrm{z}^{\prime} p_{1}^{\prime} \cdots p_{k}^{\prime} p_{\star}^{1 . . .] i[. . n} \Rightarrow b ; \operatorname{expand}\left(c_{\star}^{1 . . m}\right) \quad \mathrm{z}^{\prime}$ fresh

$\operatorname{expand}\left(\operatorname{case} p_{\star} \quad p \quad p_{\star}^{1 . .] i[. . n} \Rightarrow b ; c_{\star}^{1 . . m}\right)=$

case $p_{\star} p \mathbf{z}_{1}^{\prime} \cdots \mathbf{z}_{k}^{\prime} p_{\star}^{1 . .] i[. . n} \Rightarrow b ; \operatorname{expand}\left(c_{\star}^{1 . . m}\right) \quad \mathbf{z}_{\star}^{\prime 1 . . k}$ fresh $\quad p \neq \hat{v} \cdot \mathbf{m}\left(p_{\star}^{1 \ldots . k}\right)$

$\operatorname{other}(\bullet)=\bullet$

$\operatorname{other}\left(\right.$ case $\left.p_{\star} \hat{v} . \mathrm{m}\left(p_{\star}^{\prime 1 . . k}\right) p_{\star}^{1 . .] i[. . n} \Rightarrow b ; c_{\star}^{1 . . m}\right)=\operatorname{other}\left(c_{\star}^{1 . . m}\right)$

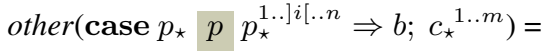

case $p_{\star} p p_{\star}^{1 . .] i[. . n} \Rightarrow b ; \operatorname{other}\left(c_{\star}^{1 . . m}\right) \quad p \neq \hat{v} \cdot \mathrm{m}\left(p_{\star}^{1 . . k}\right)$

Fig. 7. FPat Translation Rules 
sible to omit its evaluation without changing the meaning of the program. For this reason, the semantics does not cover these anomalous situations (if we included them, we could not prove our optimization correct). Any semantics for pattern matching that involves user-defined code depends on this assumption if optimized translation of matching is to preserve the meaning of programs, since we usually do not expect divergent or exception throwing programs to turn into normally terminating ones.

The assumption that extractors are @safe complements the assumptions formulated by Syme et al [11] and Okasaki [8] that informally require extractors to be side-effect free or return the same result in all execution contexts in order for optimization to work. Of course in this calculus, absence of side-effects is guaranteed by the absence of assignment.

\subsection{The Algorithm}

We define a function transform that recursively traverses expressions, rewriting any match statements it finds.

$$
\begin{aligned}
& \operatorname{transform}(\text { null }) \quad=\text { null } \\
& \operatorname{transform}(\mathrm{x}) \quad=\mathrm{x} \\
& \operatorname{transform}(\text { e.f) } \quad=\text { transform }(e) . \mathrm{f} \\
& \operatorname{transform}\left(e \cdot \mathrm{m}\left(e_{\star}^{1 . . n}\right)\right) \quad=\operatorname{transform}(e) \cdot \mathrm{m}\left(e_{\star}^{\prime}{ }_{\star}^{1 . . n}\right) \\
& \begin{array}{ll}
\operatorname{transform}(\text { throw }) & =\text { throw } \\
\operatorname{transform}(a ?\{\mathrm{x}: C \Rightarrow d\} /\{e\}) & =a^{\prime} ?\left\{\mathrm{x}: C \Rightarrow d^{\prime}\right\} /\left\{e^{\prime}\right\}
\end{array} \\
& \text { where } d^{\prime}=\operatorname{transform}(d) \\
& \text { and } e^{\prime}=\operatorname{transform}(e) \\
& \operatorname{transform}\left(e_{\star}^{1 \ldots . n} \operatorname{match}\left\{c_{\star}^{1 . . k}\right\}\right)=\operatorname{transform}\left(\operatorname{rewrite}\left(e_{\star}^{1 . . n} \operatorname{match}\left\{c_{\star}^{1 . . k}\right\}\right)\right)
\end{aligned}
$$

The transform function is then naturally extended to method definitions and class definitions. A program is translated by translating all class definitions and the top-level expression. Note that a single application of a rewrite rule takes place in one of the following contexts:

Def 1 (Target Context) A target context is defined by the following grammar:

$$
\begin{aligned}
& \xi, \zeta::=[]|\xi . \mathrm{f}| \xi \cdot \mathrm{m}\left(b_{\star}^{1 . . n}\right) \mid a \cdot \mathrm{m}\left(b_{\star}, \xi, b_{\star}{ }_{\star}^{1 . .] i[. . n}\right) \\
& |\xi ?\{\mathrm{x}: C \Rightarrow d\} /\{e\}| a ?\{\mathrm{x}: C \Rightarrow \xi\} /\{e\} \mid a ?\{\mathrm{x}: C \Rightarrow d\} /\{\xi\}
\end{aligned}
$$

By the reasoning in the next section, this rewrite preserves the meaning of the program. A subsequent call of transform performs the same for subexpressions of $a^{\prime}$, until all match expressions are translated away.

\section{Correctness}

We define a formal notion of equivalence. Recall that a substitution always satisfies $\times \sigma \equiv$ null or $\mathrm{x} \sigma \in$ Values for all $\mathrm{x} \in \operatorname{dom}(\sigma)$. We proceed in two steps, following the démarche of [14]: we define a notion of equivalence and show that it is stable under contexts. Then we show that an expression is equivalent to its translation. 


\subsection{Equivalence and Open Equivalence}

Def 2 (Equivalence) For $d$, e expressions, $f v(d)=f v(e)=\emptyset, d$ is equivalent to $e$ (written $d \approx e$ ), if both of these conditions hold: 1. for all $q$, if $d \Downarrow q$ then $e \Downarrow q$, and 2 . if $d \Uparrow$ then $e \Uparrow$.

Showing that $\approx$ is an equivalence relation is easy using (Uniqueness),(Termination). Equivalence alone is not enough for our purpose, since rewrite rules take place in context. We now define an equivalence on open terms and show it is stable under contexts.

Def 3 (Open Equivalence) For expressions $d$, e with $f v(d) \cup f v(e) \subseteq X, d$ is open-equivalent to $e$ (written $X \Vdash d \approx e$ ) if for all substitutions $\sigma$ with $X \subseteq \operatorname{dom}(\sigma)$ it holds that $d \sigma \approx e \sigma$.

Lemma 11 (Substitution preserves Equivalence) If $X \Vdash d \approx e$, then for any substitution $\sigma$ with $\operatorname{dom}(\sigma) \subseteq X$, it holds that $X \backslash \operatorname{dom}(\sigma) \Vdash d \sigma \approx e \sigma$.

Thm 2 (Congruence) If $X \Vdash d \approx e$, then $Y \Vdash \xi[d] \approx \xi[e]$ for $Y=f v(\xi[d]) \cup f v(\xi[e])$.

We now have everything we need for the correctness theorem. Since equivalence is a congruence, it is enough to show correctness of the rewrite rules.

Thm 3 (Correctness of $\llbracket \rrbracket)$ For $a \equiv a_{\star}^{1 . . n}$ match $\left\{c_{\star}^{1 . . m}\right\}$, $f v(a)=X$, typing $\Gamma \vdash a \in A$, translation $a^{\prime}=\llbracket \Gamma \vdash a \in A \rrbracket$ it holds that $X \Vdash a \approx a^{\prime}$.

Corollary 1 (Complete Algorithm) The algorithm described in Section 3.3 is correct.

Proof Consequence of the above theorem, applied sequentially to every application of a rewrite rule, and transitivity of $\approx$. For termination, observe that each match expression produced by a rewriting rule is smaller than the original match expression using the lexicographically ordered tuples $\langle i, j, k\rangle$ where $i$ is the number of non-variable input values, $j$ the number of case clauses, and $k$ the number of extractor patterns in $c_{\star}^{1 . . j}$. We observe that all chains of dependency-pairs $\left\langle\right.$ transform $(e)$, transform $\left(\right.$ rewrite $\left.\left.\left(e^{\prime}\right)\right)\right\rangle$ must thus be finite.

\section{Related Work}

Pettersson [4] and Ramsay and Scott [5] describe the same algorithm for translation match expressions to decision trees (the latter allowing heuristics other than left-to-right). Since an algebraic data type defines a closed set of constructors, different optimizations are available.

If extractors came with information that asserts that they are mutually exclusive and that they cover a fixed type, then more optimizations and incompleteness checking can be integrated. Syme, Neverov and Margetson [11] use structured names for this purpose. The authors also introduce parameterized patterns and argue for side-effect free extractors.

More generally, extractors continue a line of work that stems from views [7, 8]. The design in a functional language context that comes closest to ours is Gostanza [15]. A more detailed discussion of the literature on views is presented in [10].

Zenger and Odersky use pattern matching and algebraic datatypes in an object-oriented setting to handle the extensibility problem [16]. Liu and Myers add pattern matching to a Java like language by introducing forwards and backwards modes of evaluation [17]. 


\section{Conclusion}

We presented a formal object-oriented calculus with pattern. We proved it calculus sound, and gave an optimizing translation algorithm. We then proved the translation correct, revealing an important assumption required for correctness: that extractor patterns may not throw exceptions or diverge.

In future work, we would like to integrate convenient ways to regain incompleteness checking and further optimizations as known from algebraic data types for extractors. The Scala language offers matching on specific types (case classes) and the sealed keyword to this end. Further study is necessary to analyze how the backtracking approaches to pattern match translation can be adapted.

\section{References}

1. Burstall, R.M.: Proving properties of programs by inductive definitions. Computer Journal (1969) 41-48

2. Wadler, P.: Pattern matching, chapter 4 of Peyton Jones, Wadler 'Implementation of Functional Programming Languages" (1987)

3. Field, A., Harrison, P.: Functional Programming. Addison Wesley (1988)

4. Pettersson, M.: A term pattern-match compiler inspired by finite-automata theory. In: Proc. of International Workshop on Compiler Construction (CC). Volume Volume 641 of LNCS. (1992)

5. Scott, K., Ramsey, N.: When do match-compilation heuristics matter? Technical Report CS-2000-13, University of Virginia (2000)

6. Fessant, F.L., Maranget, L.: Optimizing pattern matching. In: Proc. of International Conference on Functional Programming(ICFP). (2001) 26-37

7. Wadler, P.: Views: A way for pattern matching to cohabit with data abstraction. In: Proc. of Principles of Programming Languages (POPL). (1987)

8. Okasaki, C.: Views for Standard ML. In: Proceedings of SIGPLAN Workshop on ML. (1998) 14-23

9. Gamma, E., Helm, R., Johnson, R., Vlissides, J.: Design Patterns. Addison-Wesley (1995)

10. Emir, B., Williams, J., Odersky, M.: Matching objects with patterns. In: Proc. of European Conference on Object-Oriented Programming (ECOOP). (2007)

11. Syme, D., Neverov, G., Margetson, J.: Combining total and ad hoc extensible pattern matching in a lightweight language extension. Technical Report MSR-TR-2007-33, Microsoft Research, Cambridge (August 2006)

12. Igarashi, A., Pierce, B., Wadler, P.: Featherweight Java. In: Proceedings of the 1999 ACM SIGPLAN Conference on Object-Oriented Programming, Systems, Languages \& Applications (OOPSLA). (1999)

13. Leroy, X., Grall, H.: Coinductive big-step operational semantics. Theoretical Computer Science ((submitted))

14. Ma, Q.: Concurrent Classes and Pattern Matching in the Join Calculus. PhD thesis, INRIARocquencourt and University Paris 7 (2005)

15. Gostanza, P.P., Pena, R., Nunez, M.M.: A new look at pattern matching in abstract data types. In: Proceedings of International Conference on Functional Programming (ICFP). (1996)

16. Zenger, M., Odersky, M.: Extensible algebraic datatypes with defaults. In: Proceedings of the International Conference on Functional Programming (ICFP). (2001)

17. Liu, J., Myers, A.C.: Jmatch: Iterable Abstract Pattern Matching for Java. In: Proceedings of 5th Internnational Symposium on Practical Aspects of Declarative Languages (PADL). (2003) 110-127 


\section{A Free, Defined Variables and Substitution}

$$
\text { Expressions } \begin{aligned}
f v(\text { null }) & =\bullet \\
f v(\text { throw }) & =\bullet \\
f v(\mathbf{x}) & =\{\mathrm{x}\} \\
f v(e . \mathrm{f}) & =f v(e) \\
f v\left(e . \mathrm{m}\left(e_{\star}^{1 . . n}\right)\right) & =f v(e) \cup \bigcup_{1 . . n} f v\left(e_{\star}\right) \\
f v\left(C\left(e_{\star}^{1 . . n}\right)\right. & =\bigcup_{1 . . n} f v\left(e_{\star}\right) \\
f v(a ?\{\mathrm{x}: C \Rightarrow e\} /\{d\}) & =f v(a) \cup f v(d) \cup f v(e) \backslash\{\mathrm{x}\} \\
f v\left(e_{\star}^{1 . . n} \mathbf{m a t c h}\left\{c_{\star}^{1 . . k}\right\}\right) & =\bigcup_{1 . . n} f v\left(e_{\star}\right) \cup \bigcup_{1 . . k} f v\left(c_{\star}\right)
\end{aligned}
$$

Case Clauses and Patterns

$$
\begin{aligned}
f v\left(\text { case } p_{\star}{ }_{\star}^{1 . . n} \Rightarrow b\right) & =f v\left(p_{\star}{ }^{1 . . n}\right) \cup f v(b) \backslash \bigcup_{1 . . n} d v\left(p_{\star}\right) \\
f v(\mathrm{x}) & =\bullet \\
f v\left(\hat{v} . \mathrm{m}\left(p_{\star}^{1 . . n}\right)\right. & =f v(\hat{v})
\end{aligned}
$$

$$
\text { Expressions } \begin{aligned}
d v(\text { null }) & =\bullet \\
d v(\text { throw }) & =\bullet \\
d v(\mathrm{x}) & =\bullet \\
d v(e . \mathrm{f}) & =d v(e) \\
d v\left(e . \mathrm{m}\left(e_{\star}^{1 . . n}\right)\right) & =d v(e) \cup \bigcup_{1 . . n} d v\left(e_{\star}\right) \\
d v\left(C\left(e_{\star}^{1 . . n}\right)\right. & =\bigcup_{1 . . n} d v\left(e_{\star}\right) \\
d v(a ?\{\mathrm{x}: C \Rightarrow e\} /\{d\}) & =d v(a) \cup d v(d) \cup d v(e) \cup\{\mathrm{x}\}
\end{aligned}
$$

Case Clauses and Patterns

$$
\begin{aligned}
d v\left(\text { case } p_{\star}^{1 . . n} \Rightarrow b\right) & =\bigcup_{1 . . n} d v\left(p_{\star}\right) \cup d v(b) \\
d v(\mathrm{x}) & =\{\mathrm{x}\} \\
d v\left(\hat{v} . \mathrm{m}\left(p_{\star}^{1 . . n}\right)\right. & =\bigcup_{1 . . n} d v\left(p_{\star}\right)
\end{aligned}
$$

$$
\begin{aligned}
f v([]) & =\bullet \\
d v([]) & =\bullet
\end{aligned}
$$


Substitution in expressions $\sigma$ :

$$
\begin{aligned}
\mathrm{x} \sigma & = \begin{cases}e & \text { if }\langle\mathrm{x}, e\rangle \in \sigma \\
\mathrm{x} & \text { otherwise }\end{cases} \\
e . \mathrm{f} \sigma & =e \sigma . \mathrm{f} \\
e . \mathrm{m}\left(e_{\star}^{1 . . n}\right) \sigma & =e \sigma \cdot \mathrm{m}\left(e_{\star} \sigma^{1 . . n}\right) \\
\text { null } \sigma & =\text { null } \\
C\left(e_{\star}^{1 . . n}\right) \sigma & =C\left(e_{\star} \sigma^{1 . . n}\right) \\
\text { throw } \sigma & =\text { throw } \\
a ?\{\mathrm{x}: C \Rightarrow b\} /\{d\} \sigma & =a \sigma ?\{\mathrm{y}: C \Rightarrow b \sigma\} /\{d \sigma\} \\
e_{\star}^{1 . . n} \operatorname{match}\left\{c_{\star}^{1 . . k}\right\} \sigma & =e_{\star} \sigma^{1 . . n} \operatorname{match}\left\{c_{\star} \sigma^{1 . . k}\right\} \\
\left(\text { case } p_{\star}^{1 . . n} \Rightarrow b\right) \sigma & =\operatorname{case} p_{\star} \bar{\sigma}^{1 . . n} \Rightarrow b \sigma
\end{aligned}
$$

Substitution in patterns $\bar{\sigma}$ :

$$
\begin{aligned}
x \bar{\sigma} & =\mathrm{x} \\
\hat{v} \cdot \mathrm{m}\left(p_{\star}{ }^{1 . . k}\right) \bar{\sigma} & =\hat{v} \sigma \cdot \mathrm{m}\left(p_{\star} \bar{\sigma}^{1 . . k}\right)
\end{aligned}
$$

\section{B Proofs for Type Soundness}

Lemma 1 (Uniqueness) For every $a$, if $a \Downarrow q$ then for all $q^{\prime}$, if $a \Downarrow q^{\prime}$ then $q=q^{\prime}$.

Proof By induction on $a \Downarrow q$ and case analysis on $q^{\prime}$.

Lemma 2 (Termination) For all $a$ and all $q$, it holds that if $a \Downarrow q$ then $a \nmid$.

Proof By induction on $a \Downarrow q$ and inversion of $a \Uparrow$. We only show (Rfld).

Case $a \equiv e . f(\mathrm{Rfld}),(\mathrm{Dfld})$ Assume $a \Downarrow q$, then by (Rfld) $e \Downarrow v$. By i.h. $e$ 你, so (Dfld) is not available. Hence $e . f$ p .

Lemma 3 (Subtypes have all Fields) If $C<: D, C \neq \operatorname{Exc}$ then fields $(C)=$ fields $(D) ; \mathrm{g}_{\star}: E_{\star}{ }^{1 . . m}$.

Proof By induction on the derivation of $C<: D$.

Case (Sobj) Then fields $(\mathrm{Obj})=\bullet$

Case (Sthr) Cannot happen

Case (Sref) Trivial

Case (Sext) Then the definition of fields is applied

Case (Stran) The i.h. is applied twice.

Lemma 4 (Subtypes have all Methods) If $C<: D, C \neq \operatorname{Exc}$ and $\operatorname{mtype}(\mathrm{m}, D)=\operatorname{an}\left(C_{\star}^{1 . . n}\right) B$, then $\operatorname{mtype}(\mathrm{m}, C)=\operatorname{an}\left(C_{\star}^{1 . . n}\right) B$. 
Proof By induction on the derivation of mtype $(\mathrm{m}, D)=a n\left(C_{\star}^{1 . . n}\right) B$ and case analysis over $C<: D$.

Case (Sobj) Cannot happen, since Obj has no methods.

Case (Sthr) Cannot happen

Case (Sref) Trivial

Case (Sext) If $C$ does not contain a definition for $\mathrm{m}$, then the definition of mtype is applied. Otherwise, class definition of $C$ is well-typed, thus override $\left(a n\left(C_{\star}^{1 . . n}\right) B, \mathrm{~m}, D\right)$ asserts that $\mathrm{mtype}(\mathrm{m}, C)=\operatorname{mtype}(\mathrm{m}, D)$.

Case (Stran) The i.h. is applied twice

\section{Lemma 5 (Subtypes yield Refined Environment)}

If $C<: D$ and $\Gamma ; D \ni p \dashv \Delta$ then $\Gamma ; C \ni p \dashv \Delta^{\prime}$ for some $\Delta^{\prime}<: \Delta$.

Proof By induction on $\Gamma ; D \ni p \dashv \Delta$

Case (TPvar) Then $\Gamma ; D \ni \mathrm{x} \dashv\{\mathrm{x}: D\}$ and we can also derive $\Gamma ; C \ni \mathrm{x} \dashv\{\mathrm{x}: C\}$. From $C<: D$ follows $\{\mathrm{x}: C\}<:\{\mathrm{x}: D\}$

Case (TPextr) Then $\Gamma ; D \ni \hat{v} \cdot \mathrm{m}\left(p_{\star}^{1 . . n}\right) \dashv \Delta_{\star}^{1 . . n}$ and subpatterns have derivations $\Gamma ; D_{\star} \ni p_{\star} \dashv \Delta_{\star}^{1 . . m}$ for some casefield types $D_{\star}{ }^{1 . . m}$. The expected type is not used for typing the subpatterns, thus the subderivations can be reused as is, yielding $\Gamma ; C \ni \hat{v} \cdot \mathrm{m}\left(p_{\star}^{1 . . n}\right) \dashv$ $\Delta_{\star}^{1 \ldots . n}$.

Lemma 6 (Refined Environment preserves Typing) If $C_{\star}<: D_{\star}^{1 . . n}$ and $\Gamma, \mathrm{x}_{\star}: D_{\star}^{1 . . n} \vdash e \in$ $B$ then $\Gamma, \mathrm{x}_{\star}: C_{\star}^{1 . . n} \vdash e \in A$ for $A<: B$.

Proof By straightforward induction on $\Gamma, \mathrm{x}_{\star}: D_{\star}^{1 . . n} \vdash e \in B$.

Lemma 7 (Weakening) If $\Gamma \vdash d \in D$ and $\mathrm{x} \notin f v(d)$, then $\Gamma, \mathrm{x}: C \vdash d \in D$. for any $C$.

Proof Straightforward induction on $\Gamma \vdash d \in D$.

Lemma 8 (Substitution Lemma) If $\Gamma, x_{\star}: B_{\star}^{1 . . n} \vdash b \in D$ and $\bullet \vdash a_{\star} \in A_{\star}^{1 . . n}$ for $A_{\star}<: B_{\star}^{1 . . n}$, $a_{\star} \in$ Values $\cup\{$ null $\}$ then $\Gamma \vdash b\left\{\mathrm{x}_{\star} \mapsto a_{\star}^{1 . . n}\right\} \in C$, for $C<: D$.

Proof By induction on the derivation of $\Gamma, \mathrm{x}_{\star}: B_{\star}^{1 . . m} \vdash b \in D$. Let $\sigma=\left\{\mathrm{x}_{\star} \mapsto a_{\star}^{1 . . n}\right\}$ and $\Delta=\Gamma, \mathrm{x}_{\star}: B_{\star}^{1 . . m}$.

$$
\text { Case (Tvar) } b \equiv \mathrm{x}
$$

i) If $\mathrm{x}=\mathrm{x}_{i}$ for some $i$, then $\mathrm{x} \sigma=a_{i}$ with $\Gamma \vdash a_{i} \in A_{i}$ and $A_{i}<: B_{i}$ by assumption, (Weakening).

ii) Otherwise, $x \sigma=x$ and rule (Tvar).

Case (Tthr), (Tnul) trivial because $b \sigma \equiv b$

Case (Tfld) $b \equiv e$.f We have $\Delta \vdash e \in E$ and i.h. yields $\Gamma \vdash e \sigma \in E^{\prime}$ for $E^{\prime}<: E$. By (Subtypes have all fields) we have fields $\left(E^{\prime}\right)=$ fields $(E) ; g_{\star}: D_{\star}^{1 \ldots . m}$ and (Tfld) finishes the case. 
Case (Tinvk) $b \equiv e \cdot \mathrm{m}\left(e_{\star}^{1 . . n}\right)$ We have $\Delta \vdash e \in E$ and $\mathrm{mtype}(\mathrm{m}, E)=a n\left(C_{\star}^{1 . . n}\right) D$. The i.h. yields $\Gamma \vdash e \sigma \in E^{\prime}$ for $E^{\prime}<: E$. We also have $\Delta \vdash e_{\star} \in E_{\star}^{1 . . n}$ for $E_{\star}<: C_{\star}^{1 . . n}$ and i.h. yields $\Gamma \vdash e_{\star} \sigma \in E^{\prime}{ }_{\star}^{1 . . n}$ for $E_{\star}^{\prime}<: E_{\star}{ }_{\star}^{1 . . n}$. By (Subtypes have all methods), we know $\operatorname{mtype}\left(\mathrm{m}, E^{\prime}\right)=\operatorname{mtype}(\mathrm{m}, E)$. Transitivity of $<:$ and rule (Tinvk) finishes the case.

Case (Tnew) $b \equiv C\left(e_{\star}^{1 . . n}\right)$ We have fields $(C)=C_{\star}^{1 . . n}$ and $\Delta \vdash e_{\star} \in E_{\star}^{1 . . n}$ with $E_{\star}<: C_{\star}{ }^{1 . . n}$. The i.h. yields $\Gamma \vdash e_{\star} \sigma \in E_{\star}^{\prime 1 . . n}$ for $E_{\star}^{\prime}<: E_{\star}{ }_{\star}^{1 \ldots . n}$. Transitivity of $<:$ and (Tnew) finish the case.

Case (Ttst) $b \equiv a ?\{\mathrm{x}: C \Rightarrow d\} /\{e\}$ We have $\Delta \vdash a \in A, \Delta \vdash d \in E_{1}$, and $\Delta \vdash e \in$ $E_{0}$ and $E_{\star}<: D^{0,1}$. The i.h. yields $\Gamma \vdash a \sigma \in A^{\prime}, \Gamma \vdash d \sigma \in E_{1}^{\prime}$, and $\Gamma \vdash e \sigma \in E_{0}^{\prime}$ with $A^{\prime}<: A$, $E_{1}^{\prime}<: E_{1}$ and $E_{0}^{\prime}<: E_{0}$. Transitivity of $<:$ and (Ttst) finishes the case.

Case (Tmch) $b \equiv e_{\star}^{1 . . n}$ match $\left\{c_{\star}^{1 . . m}\right\}$ We have $\Delta \vdash e_{\star} \in C_{\star}^{1 . . n}$ and i.h. yields $\Gamma \vdash e_{\star} \sigma \in C^{\prime}{ }_{\star}^{1 . . n}$ for $C_{\star}^{\prime}<: C_{\star}{ }_{\star}^{1 \ldots . n}$.

For each $j \in 1 . . m$, let $c_{j} \equiv$ case $p_{\star}^{1 . . n} \Rightarrow b_{j}$ (we omit the extra $j$ index for patterns). We have a case typing $\Delta ; C_{\star}^{1 . . m} \vdash c_{j} \in D_{j}$ via $\Delta ; C_{\star} \ni p_{\star} \dashv \Sigma_{\star}^{1 \ldots . n}$ and $\Delta, \Sigma_{\star}^{1 . . n} \vdash b \in D_{j}$.

By (Subtypes yield Refined Environment), we get $\Delta ; C_{\star}^{\prime} \ni p_{\star} \dashv \Sigma_{\star}^{1 . . n}$ for $\Sigma_{\star}^{\prime}<: \Sigma_{\star}^{1 . . n}$. By (Refined Environment preserves Typing) we get $\Gamma, \Sigma_{\star}^{\prime}{ }_{\star}^{1 . . n} \vdash b_{j} \in D_{j}^{\prime}$ for $D_{j}^{\prime}<: D_{j}$. Applying the i.h. yields $\Gamma, \Sigma_{{ }_{\star}}{ }^{1 . . n} \vdash b_{j} \sigma \in D_{j}^{\prime \prime}$ for $D_{j}^{\prime \prime}<: D_{j}^{\prime}$.

For the combined lubs, we have $\bigsqcup D_{\star}^{\prime \prime 1 . . m}<: \bigsqcup D_{\star}^{1 \ldots . m}$, and rule (Tmch) finishes the case.

\section{Lemma 9 (Preservation)}

If $a \Downarrow q$ and $\bullet \vdash a \in C$, then $\bullet \vdash q \in C^{\prime}$ for some $C^{\prime}<: C$.

Proof For $q \equiv$ throw and $q \equiv$ null, rules (Tthr) and (Tnul) yield the proof. Otherwise, induction on $a \Downarrow v$.

Case (Rfld) $a \equiv e . f_{i}$

The premises of (Tfld) are $\bullet \vdash e \in C_{0}$ and fields $\left(C_{0}\right)=\mathrm{f}_{\star}: C_{\star}{ }_{\star}^{1 . . m}$ with $C=C_{i}$.

We have $e \Downarrow D\left(w_{\star}^{1 . . n}\right)$ and $v=w_{i}$.

By i.h. $\bullet \vdash D\left(w_{\star}^{1 . . n}\right) \in D$ for $D<: C_{0}$.

By Lemma (Subtypes have all fields), we obtain $m \leq n$ and $f_{i} \in$ fields $(D)$.

Finally, from $\bullet \vdash D\left(w_{\star}^{1 . . n}\right) \in D$ and rule (Tnew) we know $\bullet \vdash w_{i} \in E_{i}$ with $E_{i}<: C_{i}$.

Case (Rinvk) $a \equiv e \cdot \mathrm{m}\left(e_{\star}^{1 \ldots . n}\right)$

The premises of $($ Tinvk $)$ are $\Gamma \vdash e \in E, \quad \operatorname{mtype}(\mathrm{m}, E)=\operatorname{an}\left(C_{\star}^{1 . . n}\right) C_{0}, \quad \bullet \vdash e_{\star} \in C_{\star}^{1 \ldots . n}$ and $\bullet \vdash e_{0} \in C$.

Then $e \Downarrow D\left(w_{\star}^{1 . . m}\right), e_{\star} \Downarrow v_{\star}^{1 \ldots . n}, \operatorname{mbody}(D, \mathrm{~m})=\left(\mathrm{x}_{\star}^{1 . . n}\right) e_{0}$ and a substitution $\sigma=$ $\left\{\right.$ this $\left.\mapsto D\left(w_{\star}^{1 . . m}\right), x_{\star} \mapsto v_{\star}^{1 . . n}\right\}$, which makes the body evaluates as $e_{0} \sigma \Downarrow v$.

Applying the i.h. for the receiver yields $D<: E$.

By (Subtypes have all Methods) we get $\mathrm{mtype}(D, \mathrm{~m})=\operatorname{mtype}(\mathrm{m}, E)$.

Applying the i.h. for the arguments yields $\bullet \vdash v_{\star} \in C_{\star}^{\prime 1 . . n}$ for $C_{\star}^{\prime}<: C_{\star}^{1 . . n}$.

By (Substitution Lemma) we get $\bullet \vdash e_{0} \sigma \in C^{\prime}$ for $C^{\prime}<: C$.

Applying the i.h. for the body then yields $\bullet \vdash v \in C^{\prime}$.

Case (Rnew) $a \equiv D\left(w_{\star}^{1 . . n}\right)$ then $a \Downarrow a$, and $D<: D$ by (Sref).

Case (Rcst) $a \equiv e ?\{\mathrm{x}: C \Rightarrow b\} /\{d\}$

We have $e \Downarrow D\left(w_{\star}^{1 . . n}\right), D<: C$ and $b\left\{\mathrm{x} \mapsto D\left(w_{\star}^{1 . . n}\right)\right\} \Downarrow v$. 
Using typing premises from (Ttst), we apply the (Substitution Lemma) and then the i.h.

Case (Rskp) $a \equiv e ?\{\mathrm{x}: C \Rightarrow b\} /\{d\}$

We have $e \Downarrow D\left(w_{\star}^{1 . . n}\right), D \not: C$ and $d \Downarrow v$.

Using typing premises from (Ttst), we apply the i.h. to $d$, yielding $\bullet \vdash d \in C^{\prime}$.

Case (Rmch) $a \equiv e_{\star}^{1 . . m}$ match $\left\{c_{\star}^{1 . . . l}\right\}$. Let $i$ be the index of the matching case.

The premises of (Tmch) include $\bullet \vdash e_{\star} \in C_{\star}^{1 . . m}$ and case typing $\bullet ; C_{\star}^{1 . . m} \vdash c_{i} \in D_{i}$ for $D_{i}<: C$.

The case typing has premises $\bullet ; C_{\star} \ni p_{\star} \dashv \Sigma_{\star}^{1 . . n}$ and $\bullet ; \Sigma_{\star}^{1 . . m} \vdash b \in D_{i}$ where $c_{i} \equiv$ case $p_{\star} \Rightarrow b$.

We have $e_{\star} \Downarrow v_{\star}^{1 . . m}, v_{\star}^{1 . . m} \curvearrowright p_{\star} \dashv \sigma_{\star}$ and $b \sigma_{\star} \Downarrow v$.

Applying the induction hypothesis to $e_{\star}$ yields $\bullet \vdash v_{\star} \in C_{\star}^{\prime 1 \ldots n}$ for $C_{\star}^{\prime}<: C_{\star}^{1 \ldots . n}$.

By (Substitution Lemma), $\bullet \vdash b \sigma_{\star} \in D_{i}^{\prime}$ with $D_{i}^{\prime}<: D_{i}$.

Applying the i.h. yields $\bullet \vdash v \in D_{i}^{\prime \prime}$ with $D_{i}^{\prime \prime}<: D_{i}^{\prime}$.

This yields the desired type $C^{\prime}=D_{i}^{\prime \prime}<: \bigsqcup D_{\star}^{11 . m}=C$, by transitivity of $<$ : and properties of the lub.

\section{Lemma 10 (Progress)}

If $\bullet-a \in C$ and $a \Downarrow q$ for all $q$, then $a \Uparrow$.

Proof By coinduction and case analysis over $a$. We recall the principle of coinduction: In order to show that $R=\{a \mid$ for all $q$. $a \Downarrow q$ and $\bullet \vdash a \in C\}$ is included in $\Uparrow$, it suffices to show that $R$ is preserved by the divergence rules. This means, if we replaced each assertion $a \Uparrow$ with $a \in R$ and assumed that the conclusion holds, we have to be able to show that the premises hold as well. To do this, we need proofs for $e \nVdash$ for the subexpressions $e$ of $a$. These can be obtained from inversion of "blocked" evaluation rules.

Case $a \in\{$ throw, null $\}$ and $a \equiv \mathrm{x}$ are not interesting, since $a \notin R$

Case $a \equiv e_{0} . f$ and (Rfld), (Cfld) are blocked. By $\bullet \vdash a \in C$ and (Tfld), we also have

$\bullet \vdash e_{0} \in C_{0}$. Thus, either

i) $e_{0} \Downarrow q_{0}$ for any $q_{0}$. This amounts to $e_{0} \in R$ and shows that (Dfld) preserves $R$.

ii) $e_{0} \Downarrow$ throw or $e_{0} \Downarrow$ null , but this contradicts (Cfld) blocked.

iii) $e_{0} \Downarrow D\left(w_{\star}^{1 . . n}\right)$ but by (Preservation) and (Subtypes have all fields), this contradicts (Rfld) blocked.

Case $a \equiv e_{0} \cdot \mathrm{m}\left(e_{\star}^{1 . . n}\right)$ and (Rinvk),(Crcv) and (Carg) are blocked. By $\bullet \vdash a \in C$ and (Tinvk), we also have $\bullet \vdash e_{0} \in C_{0}, \bullet \vdash e_{\star} \in C_{\star}^{1 \ldots . n}$, mtype $\left(\mathrm{m}, C_{0}\right)=\left(D_{\star}^{1 . . n}\right)$ and $C_{\star}<: D_{\star}^{1 . . . n}$.

Thus, either

i) $e_{0} \Downarrow q_{0}$ for any $q_{0}$. This amounts to $e_{0} \in R$ and shows that (Drcv) preserves $R$.

ii) $e_{0} \Downarrow$ throw or $e_{0} \Downarrow$ null but this contradicts (Crcv) blocked.

iii) $e_{0} \Downarrow D\left(w_{\star}^{1 . . n}\right)$. By (Preservation), $D<: C_{0}$ and by (Subtypes have all methods), $\operatorname{mbody}(\mathrm{m}, D)=$ $\left(\mathrm{x}_{\star}^{1 . . n}\right) b$. We can distinguish further

a) There exists $i$ with $e_{\star} \Downarrow v_{\star}^{1 . . i-1}$ and $e_{i} \Downarrow q_{0}$ for any $q_{0}$. Then $e_{i} \in R$ and (Darg) preserves $R$. 
b) There exists $i$ with $e_{\star} \Downarrow v_{\star}^{1 . . i-1}$ and $e_{i} \Downarrow$ throw, but this contradicts (Carg) blocked

c) $e_{\star} \Downarrow v_{\star}^{1 . . n}$ and (Preservation) yields $\bullet \vdash v_{\star} \in D_{\star}^{1 . . n}$ for $D_{\star}<: C_{\star}^{1 . . n}$. Then let $\sigma=\left\{\right.$ this $\left.\mapsto D\left(w_{\star}^{1 . . m}\right) \times_{\star} \mapsto v_{\star}^{1 . . n}\right\}$ and consider $b \sigma$. Either

1. $b \sigma \nVdash q$ for any $q$. This amounts to $b \sigma \in R$ and shows that (Dinvk) preserves $R$.

2. $b \sigma \Downarrow q$, but this contradicts (Rinvk) blocked.

Case $a \equiv C\left(e_{\star}^{1 . . n}\right)$ and (Rnew), (Cnew) are blocked. By $\bullet \vdash a \in C$ and (Tnew), we also have $\bullet \vdash e_{\star} \in A_{\star}{ }^{1 . . n}$, fields $(C)=B_{\star}^{1 . . n}$ and $A_{\star}<: B_{\star}{ }_{\star}^{1 . . n}$. Thus, either

i) there exists $i$ with $e_{\star} \Downarrow v_{\star}^{1 . . i-1}$ and $e_{i} \Downarrow q$ for any $q$. Then $e_{i} \in R$ and (Dnew) preserves $R$

ii) there exists $i$ with $e_{\star} \Downarrow v_{\star}^{1 . . i-1}$ and $e_{i} \Downarrow$ throw, but this contradicts (Cnew) blocked.

iii) $e_{\star} \Downarrow v_{\star}^{1 . . n}$, but this contradicts (Rnew) blocked.

Case $a \equiv e ?\{\mathrm{x}: C \Rightarrow b\} /\{d\}$ and (Rcst),(Rskp),(Ctst) are blocked. By $\bullet \vdash a \in C$ and (Ttst), we have all premises of the rule (Ttst). Thus either

i) $e \nVdash q$ for any $q$, then $e \in R$ and (Dtst) preserves $R$.

ii) $e \Downarrow$ throw, but this contradicts (Ctst) blocked.

iii) $e \Downarrow D\left(v_{\star}^{1 \ldots . n}\right)$. There are several subcases to consider:

a) $D<: C$, and for $\sigma=\left\{\mathrm{x} \mapsto D\left(v_{\star}^{1 . . n}\right)\right\}, b \sigma \nVdash q$ for any $q$. Then $b \sigma \in R$ and (Dcst) preserves R.

b) $D<: C$, and for $\sigma=\left\{\mathrm{x} \mapsto D\left(v_{\star}^{1 . . n}\right)\right\}, b \sigma \nVdash$ throw but this contradicts (Rcst) blocked.

c) $D \nless: C$, and $c \sigma \nVdash q$ for any $q$. Then $c \sigma \in R$ and (Dskp) preserves R.

Case $a \equiv e_{\star}^{1 . . n}$ match $\left\{c_{\star}^{1 . . m}\right\}$ and (Rmch),(Cmch) are blocked.

By $\bullet \vdash a \in C$ and (Tmch), we have $\bullet \vdash e_{\star} \in A_{\star}{ }_{\star}^{1 . . n}$, for all $j$ a case typing $\bullet ; A_{\star}{ }^{1 . . n} \vdash$ $c_{j} \in D_{j}$, and for each body $b_{j}$ a typing $\Delta_{j} \vdash b_{j} \in D_{j}$.

Thus, either

i) there exists $i$ with $e_{\star} \Downarrow v_{\star}^{1 . . i-1}$ and $e_{i} \Downarrow q$ for any $q$. Then $e_{i} \in R$ and (Dmch) preserves $R$.

ii) there exists $i$ with $e_{\star} \Downarrow v_{\star}^{1 . . i-1}$ and $e_{i} \Downarrow$ throw or $e_{i} \Downarrow$ null, but this contradicts (Cmch) blocked.

iii) $e_{\star} \Downarrow v_{\star}^{1 . . n}$. Then we distinguish these cases:

a) if all cases reject, this contradicts that the last case always accepts.

b) There exists an $i$ such that $\forall j<i . v_{\star}^{1 . . n} ; c_{j} \Downarrow$ reject, $c_{i}=$ case $p_{\star}^{1 . . n} \Rightarrow b$ and $v_{\star} \curvearrowright p_{\star} \dashv \sigma_{\star}^{1 . . n}$. Then, either

1) $b \sigma_{\star}^{1 . . n} \nVdash q$ for any $q$, then $b \sigma_{\star}^{1 . . n} \in R$ and (Dbdy), (Dcase) preserve $R$

2) $b \sigma_{\star}^{1 . . n} \Downarrow q$, which contradicts (Rmch) blocked.

Thus, $R$ is preserved by all divergence rules, so $R \subseteq \Uparrow$.

Thm 1 (Type Soundness)

If $\bullet \vdash a \in C$ then either $a \Uparrow$ or $a \Downarrow q$ for some $q$ with $\bullet \vdash q \in C^{\prime}, C^{\prime}<$ :C. 
Proof Consequence of (Progress) and (Termination).

\section{Proofs for Correctness}

Def 1 (Equivalence) For $d$, e expressions, $f v(d)=f v(e)=\emptyset, d$ is equivalent to $e$ (written $d \approx e$ ), if both of these conditions hold: 1. for all $q$, if $d \Downarrow q$ then $e \Downarrow q$, and 2 . if $d \Uparrow$ then $e \Uparrow$.

Def 2 (Open Equivalence) For expressions $d$, e with $f v(d) \cup f v(e) \subseteq X, d$ is open-equivalent to $e$ (written $X \Vdash d \approx e$ ) if for all substitutions $\sigma$ with $X \subseteq \operatorname{dom}(\sigma)$ it holds that $d \sigma \approx e \sigma$.

Def 3 (Target Context) A target context is defined by the following grammar:

$$
\begin{aligned}
\xi, \zeta:: & =[]|\xi \cdot f| \xi \cdot \mathrm{m}\left(b_{\star}^{1 . . n}\right) \mid a \cdot \mathrm{m}\left(b_{\star}, \xi, b_{\star}^{1 . .] i[. . n}\right) \\
& |\xi ?\{\mathrm{x}: C \Rightarrow d\} /\{e\}| a ?\{\mathrm{x}: C \Rightarrow \xi\} /\{e\} \mid a ?\{\mathrm{x}: C \Rightarrow d\} /\{\xi\}
\end{aligned}
$$

Lemma 11 (Substitution preserves Equivalence) If $X \Vdash d \approx e$, then for any substitution $\sigma$ with $\operatorname{dom}(\sigma) \subseteq X$, it holds that $X \backslash \operatorname{dom}(\sigma) \Vdash d \sigma \approx e \sigma$.

Proof. Let $X \Vdash d \approx e$ and $\sigma$ be a substitution. We have to show that for any substitution $\rho$ with $\operatorname{dom}(\rho)=X \backslash \operatorname{dom}(\sigma)$, it holds that $(d \sigma) \rho \approx(e \sigma) \rho$. Considering that substitution is associative, this amounts to $d(\sigma \rho) \approx e(\sigma \rho)$. We observe that $\sigma \rho$ is a substitution with $X=\operatorname{dom}(\sigma \rho)$, and the equivalence follows from $X \Vdash d \approx e$.

The following definition can be used to show that a rewrite rule takes a typed expression to another typed expression.

Def 4 (Target Context Typing) A context is assigned a type $[C] D$ according to the following typing rule.

$$
\frac{\Gamma, \mathrm{z}: C \vdash \xi[\mathrm{z}] \in D \quad \mathrm{z} \text { fresh }}{\Gamma \vdash \xi \in[C] D}
$$

By the (Substitution Lemma) it is easy to see, that if $\Gamma \vdash \xi \in[C] D$ and $\Gamma \vdash a \in B$ for $B<: C$, then $\Gamma \vdash \xi[a] \in D^{\prime}$ for $D^{\prime}<: D$.

Thm 2 (Congruence) If $X \Vdash d \approx e$, then $Y \Vdash \xi[d] \approx \xi[e]$ for $Y=f v(\xi[d]) \cup f v(\xi[e])$.

Proof By induction on $\xi$. For each context shape, we consider possible terminating and divergent computations under a value substitution $\sigma$.

Case $\xi \equiv[]$ then $Y=X$ and $X \Vdash d \approx e$ by assumption.

Case $\xi \equiv \zeta . f$

i) $(\zeta[d]$. f) $\sigma \Downarrow$ throw by (Cfld). Then, we have $\zeta[d] \sigma \Downarrow$ throw or $\zeta[d] \sigma \Downarrow$ null. By i.h., we obtain $\zeta[e] \sigma \Downarrow$ throw (resp. $\zeta[e] \sigma \Downarrow$ null) and $(\zeta[e] . f) \sigma \Downarrow$ throw by (Cfld).

ii) $\left(\zeta[d]\right.$. f) $\sigma \Downarrow w$ by rule (Rfld). Then, we have $\zeta[d] \sigma \Downarrow C\left(v_{\star}^{1 . . n}\right)$ and f: $D \in$ fields $(C)$. By the i.h., we obtain $\zeta[e] \sigma \Downarrow C\left(v_{\star}^{1 . . n}\right)$ and $(\zeta[e] . f) \sigma \Downarrow w$ by (Rfld).

iii) $(\zeta[d] . f) \sigma \Uparrow$ by (Dfld). Then $\zeta[d] \sigma \Uparrow$, by i.h. $\zeta[e] \sigma \Uparrow$ and $(\zeta[e] . f) \sigma \Uparrow$ by (Dfld) 


$$
\text { Case } \xi \equiv \zeta \cdot \mathrm{m}\left(b_{\star}^{1 . . n}\right)
$$

i) $\left(\zeta[d] . \mathrm{m}\left(b_{\star}{ }_{\star}^{1 . . n}\right)\right) \sigma \Downarrow$ throw by (Crcv). Then $\zeta[d] \sigma \Downarrow$ throw or $\zeta[d] \sigma \Downarrow$ throw. By i.h. $\zeta[e] \sigma \Downarrow$ throw (resp. $\zeta[e] \sigma \Downarrow$ null) and $\left(\zeta[e] . \mathrm{m}\left(b_{\star}{ }_{\star}^{1 . . n}\right)\right) \sigma \Downarrow$ throw by (Crcv).

ii) $\left(\zeta[d] . \mathrm{m}\left(b_{\star}^{1 . . n}\right)\right) \sigma \Downarrow$ throw by (Carg). Then $b_{i} \sigma \Downarrow$ throw or $b_{i} \sigma \Downarrow$ throw and $\zeta[e] . \mathrm{m}\left(b_{\star}^{1 . . n}\right) \Downarrow$ throw by (Carg).

iii) $\left(\zeta[d] . \mathrm{m}\left(b_{\star}{ }_{\star}^{1 . . n}\right)\right) \sigma \Downarrow q$ by (Rinvk). Then $\zeta[d] \sigma \Downarrow C\left(v_{\star}^{1 . . m}\right)$. By i.h., $\zeta[e] \sigma \Downarrow C\left(v_{\star}^{1 \ldots . m}\right)$ and by (Rinvk), $\zeta[e] \cdot \mathrm{m}\left(b_{\star}^{1 \ldots . n}\right) \Downarrow q$.

Case $\xi \equiv a . \mathrm{m}\left(b_{\star}, \zeta, b_{\star}^{1 . .] i[. . n}\right)$

This case is similar to the preceding case.

Case $\xi \equiv \zeta ?\{\mathrm{x}: E \Rightarrow a\} /\{b\}$

i) $(\zeta[d] ?\{\mathrm{x}: E \Rightarrow a\} /\{b\}) \sigma \Downarrow$ throw by (Ctst). Then $\zeta[d] \sigma \Downarrow$ throw. By i.h. $\zeta[e] \sigma \Downarrow$ throw and $(\zeta[e] ?\{\mathrm{x}: E \Rightarrow a\} /\{b\}) \sigma \Downarrow$ throw by (Ctst).

ii) $(\zeta[d] ?\{\mathrm{x}: E \Rightarrow a\} /\{b\}) \sigma \Downarrow q$ by (Rcst). Then $\zeta[d] \sigma \Downarrow E_{0}\left(v_{\star}^{1 . . n}\right)$ for some $E_{0}<: E$ and $a \sigma\left\{\mathrm{x} \mapsto E_{0}\left(v_{\star}^{1 . . n}\right)\right\} \Downarrow q$. The i.h. yields $(\zeta[e]) \sigma \Downarrow E_{0}\left(v_{\star}^{1 \ldots . n}\right)$ and $(\zeta[e] ?\{\mathrm{x}: E \Rightarrow$ $a\} /\{b\}) \sigma \Downarrow q$ by (Rcst).

iii) $(\zeta[d]$ ? $\{\mathrm{x}: E \Rightarrow a\} /\{b\}) \sigma \Downarrow q$ by (Rskp). Then we either have $\zeta[d] \sigma \Downarrow$ null or $\zeta[d] \sigma \Downarrow$ $C\left(v_{\star}^{1 . . n}\right)$ for some $C \not: E$, and $b \sigma \Downarrow q$. The i.h. yields $\zeta[e] \sigma \Downarrow$ null resp. $\zeta[e] \sigma \Downarrow$ $C\left(v_{\star}^{1 . . n}\right)$ and $(\zeta[e] ?\{\mathrm{x}: E \Rightarrow a\} /\{b\}) \sigma \Downarrow q$ by (Rskp).

Case $\xi \equiv a ?\{\mathrm{x}: E \Rightarrow \zeta\} /\{b\}$

This case is similar to the preceding case, with i.h. applied with $\sigma\{\mathrm{x} \mapsto w\}$ for (Rcst).

Case $\xi \equiv a ?\{\mathrm{x}: E \Rightarrow b\} /\{\zeta\}$

This case is similar to the preceding case.

Def 5 (Derived Form for Value Definition) The expression form val $\mathrm{x}: C=a ; d$ abbreviates $a ?\{\mathrm{x}: C \Rightarrow d\} /\{d\{\mathrm{x} \mapsto \mathbf{n u l l}\}\}$ and is typed according to the scheme

$$
\frac{\Gamma \vdash a \in A \quad \Gamma, \mathrm{x}: A \vdash d \in D \quad \Gamma \vdash d\{\mathrm{x} \mapsto \mathbf{n u l l}\} \in D^{\prime}}{\Gamma \vdash a ?\{\mathrm{x}: C \Rightarrow d\} /\{d\{x \mapsto \mathbf{n u l l}\}\} \in D} \text { (Ttst) }
$$

With (Substitution Lemma), it is easy to see that $D^{\prime}<: D$ and thus $D \sqcup D^{\prime}=D$

Lemma 12 (ValDef Equivalences) Let $b \equiv \operatorname{val} \times: A=a$; $d$ where $\Gamma \vdash a \in A$ and $\sigma$ some substitution that agrees with $\Gamma$. Then

$$
\begin{aligned}
& \text { I. If } a \sigma \Downarrow w \quad \text { then } b \sigma \approx d\{\mathrm{x} \mapsto w\} \sigma . \\
& I I . \text { If } a \sigma \Downarrow \text { null } \\
& \text { III. If } a \sigma \Downarrow \text { throw then } b \sigma \Downarrow d\{\mathrm{x} \mapsto \text { null }\} \sigma . \\
& I V \text {. If } a \sigma \Uparrow \quad \text { then } b \sigma \Uparrow .
\end{aligned}
$$


Proof (Sketch) We note that by (Substitution Lemma) and (Preservation), the type test cannot fail except for null. With this observation, I. follows from (Rcst), II. from (Rskp), III. from (Ctst) and IV. from (Drcv).

Def 6 (Translation) For $a \equiv e_{\star}^{1 . . n}$ match $\left\{c_{\star}^{1 . . m}\right\}$ with $\Gamma \vdash a \in C$, the translation $\llbracket \Gamma \vdash$ $a \in C \rrbracket$ is defined as the application of a suitable rule in Fig. 7.

Lemma 13 (Split) Let $a \equiv \mathbf{z}_{\star}^{1 . . n}$ match $\left\{c_{\star}^{1 . . m}\right\}$ with $\Gamma \vdash a \in A$, with $c_{1}=$ case $\times_{\star}^{1 . . i-1} \hat{v} \cdot \mathrm{m}\left(\pi_{\star}^{1 . . k}\right) p_{\star}^{i+1 . . n} \Rightarrow b$, and $x \operatorname{type}(\Gamma, \hat{v}, \mathrm{~m})=@ \mathbf{s a f e}(\mathrm{Obj}) C$.

For any substitution $\sigma$ it holds that :

I. $\hat{v} . \mathrm{m}\left(\mathrm{z}_{i}\right) \sigma \Downarrow w \quad$ implies $a \sigma \approx\left(\mathbf{z}_{\star}, \mathbf{z}_{i}, \mathbf{w}_{\star}^{1 . . k}, \mathbf{z}_{\star}^{1 . .] i[. . n} \operatorname{match}\left\{\operatorname{expand}_{\hat{v} . m}\left(c_{\star}^{1 . . m}\right)\right\}\right) \sigma$ II. $\hat{v} \cdot \mathrm{m}\left(\mathrm{z}_{i}\right) \sigma \Downarrow$ null implies $a \sigma \approx\left(\mathbf{z}_{\star}^{1 . . n}\right.$ match $\left\{\right.$ other $\left.\left._{\hat{v} . m}\left(c_{\star}^{1 . . m}\right)\right\}\right) \sigma$

Proof $I$. Let $a^{\prime} \equiv\left(\mathrm{z}_{\star}, \mathrm{z}_{i}, \mathrm{w}_{\star}^{1 . . k}, \mathrm{z}_{\star}^{1 . .] i[. . n} \operatorname{match}\left\{\operatorname{expand}_{\hat{v} . m}\left(c_{\star}{ }_{\star}^{1 . . m}\right)\right\}\right) \sigma$. Terminating computation $a \sigma \Downarrow q$ can only happen through (Rmch).

We show pattern acceptance and rejection coincides in $a$ and $a^{\prime}$.

Let $z_{\star} \sigma^{1 . . n} ; c_{j} \sigma \Downarrow$ reject. Then acceptance as well as rejection was established to the left of column $i$, in column $i$, or to the right of column $i$ of the original match in $a$.

Patterns to the left of $i$ are not changed by expand. Patterns to the right of $i$ are merely moved to index $i+k$, but test the same input values.

- If in clause $c_{j}$ a pattern in column $i$ is of the form $\hat{v} \cdot \mathrm{m}\left(\pi_{\star}^{\prime 1 . . k}\right)$ for some $\pi_{\star}^{\prime 1 . . k}$, the function expand lifts patterns $\pi_{\star}^{\prime 1 . . k}$ appear in $c_{j}^{\prime}$, to be matched against $w_{\star}^{1 . . k}$.

The same derivations for acceptance and rejection can be reused: whenever acceptance by (mextr) is derived with $w_{\star} \curvearrowright \pi_{\star}^{\prime} \dashv \rho_{\star}^{1 . . k}$ the corresponding $\rho_{\star}^{1 . . k}$ are also obtained in $c_{j}^{\prime}$. Moreover, whenever rejection is derived through (rchild), then $w_{h} \curvearrowright \pi_{h}^{\prime} \dashv$ reject for some $h$ can be also be derived in $c_{j}^{\prime}$. The additional variable pattern at position $i$ does not affect the outcome, since it was chosen fresh.

- If in clause $c_{j}$, a pattern with a different extractor appears in column $i$, then the outcome is obviously the same. The additional variable patterns in columns $i+1 . . i+k$ act as dummy patterns for discarded input values $w_{\star}^{1 . . k}$.

Divergent computation $a \sigma \Uparrow$ is only derivable with (Dcase), and (Dbdy). By the same reasoning as above, patterns in $c_{j}^{\prime}$ have the same acceptance/rejection behavior as those in $c_{j}$. So the matching case $c_{i}$ in (Dbdy) produces the same substitution $\rho$ that makes the body diverge.

Proof II. By (rnull), no case $c_{j}$ that contains $\hat{v} \cdot \mathrm{m}\left(\pi_{\star}^{\prime 1 . . k}\right)$ at column $i$ can accept input values $z_{\star}^{11 . n} \sigma$. By condition "@safe" divergent patterns and exceptions in patterns are ruled out, and omitting these clauses does not alter the behavior of $a$. Thus, $a^{\prime}$ contains exactly those cases that still need to be tested for acceptance or rejection (with at least one case always accepting, by convention). Thus, all terminating, divergent and exception-throwing computation in $a$ is simulated by a corresponding computation in $a^{\prime}$.

Thm 3 (Correctness of $\llbracket \rrbracket)$ For $a \equiv a_{\star}^{1 . . n}$ match $\left\{c_{\star}^{1 . . m}\right\}, f v(a)=X$, typing $\Gamma \vdash a \in A$, translation $a^{\prime}=\llbracket \Gamma \vdash a \in A \rrbracket$ it holds that $X \Vdash a \approx a^{\prime}$. 
Proof We make a case distinction on $\llbracket \Gamma \vdash a \in C \rrbracket$. Let $\sigma$ be a substitution that closes $a$.

Case (Tmp) Let $\sigma$ be a substitution with $X \subseteq \operatorname{dom}(\sigma)$. Then (ValDef Equivalences) yields the desired result.

Case (Var) $a=\mathrm{z}_{\star}^{1 . . n} \operatorname{match}\left\{c_{\star}^{1 . . m}\right\}$ and $a^{\prime}=b\left\{\mathrm{x}_{\star} \mapsto \mathrm{z}_{\star}^{1 . . n}\right\}$

$$
\text { where } c_{1}=\text { case } x_{\star}^{1 . . n} \Rightarrow b
$$

Since $z_{\star} \sigma \in$ Values $\cup\{$ null $\}$, we can ignore (Cmch),(Dmch). By (mcase) and (mvar), the first row matches, yielding $\rho=\{\mathrm{x} \mapsto \mathrm{z} \sigma\}$. We then exploit that $b \rho \equiv b\left\{\mathrm{x}_{\star} \mapsto \mathrm{z}_{\star}^{1 \ldots n}\right\} \sigma$

i) $a \sigma \Downarrow q$ by (Rmch). Then $b \rho \Downarrow q$ thus $b\left\{\mathrm{x}_{\star} \mapsto \mathrm{z}_{\star}^{1 . . n}\right\} \sigma \Downarrow q$

ii) $a \sigma \Uparrow$ by (Dcase),(Dbdy). Then $b \rho \Uparrow$ thus $b\left\{\mathrm{x}_{\star} \mapsto \mathrm{z}_{\star}^{1 \ldots . n}\right\} \sigma \Uparrow$

Case (Mix) $a=\mathrm{z}_{\star}^{1 . . n}$ match $\left\{c_{\star}^{1 . . m}\right\}$ and $\hat{v} \cdot \mathrm{m}\left(\mathrm{z}_{i}\right) ?\{\mathbf{x}: B \Rightarrow d\} /\{e\}$

where $c_{1}=$ case $\times_{\star}^{1 . . i-1} \hat{v} \cdot \mathrm{m}\left(p_{\star}^{1 . . k}\right) p_{\star}^{i+1 . . n} \Rightarrow b$ and $d, e$ as described in Figure 7.

Since $z_{\star} \sigma \in$ Values $\cup\{$ null $\}$, we can ignore (Cmch),(Dmch). We distinguish two cases.

- $\hat{v} \cdot \mathrm{m}\left(\mathbf{z}_{i}\right) \sigma \Downarrow w$. Then by (Preservation), (Rcst), we get $a^{\prime} \sigma \approx d \sigma$. We finish the case with Lemma Split and transitity of $\approx$.

- $\hat{v} \cdot \mathrm{m}\left(\mathrm{z}_{i}\right) \sigma \Downarrow$ null. Then by (Rskp), $a^{\prime} \sigma \approx e \sigma$. We finish the case with Lemma Split and transitity of $\approx$.

\section{A Simplistic Approximation of Safe Methods}

A method can be proven @safe, if its body is in the restricted set of "safe expressions".

$$
\begin{aligned}
& \text { Safe Expressions } \\
& \frac{\mathrm{x} \in \operatorname{dom}(\Gamma)}{\Gamma \vdash \mathrm{x} \operatorname{not} \text { null }} \quad \frac{\Gamma, \mathrm{x}: C \vdash b \operatorname{not} \text { null } \quad \Gamma \vdash d \text { not null }}{a ?\{\mathrm{x}: C \Rightarrow b\} /\{e\} \text { not null }} \\
& \overline{\Gamma \vdash \text { null safe }} \frac{\Gamma \vdash e \text { not null } \quad \Gamma \vdash e \text { safe }}{\Gamma \vdash e . f \text { safe }} \quad \frac{\Gamma \vdash a \text { safe } \quad \Gamma, \mathrm{x}: C \vdash b \text { safe } \quad \Gamma \vdash e \text { safe }}{a ?\{\mathrm{x}: C \Rightarrow b\} /\{e\} \text { safe }}
\end{aligned}
$$




\section{E The complete transform function}

$$
\begin{aligned}
& \operatorname{transform}(\text { null }) \quad=\text { null } \\
& \operatorname{transform}(\mathrm{x}) \quad=\mathrm{x} \\
& \operatorname{transform}(\text { e.f }) \quad=\operatorname{transform}(e) . \mathrm{f} \\
& \operatorname{transform}\left(e . \mathrm{m}\left(e_{\star}^{1 . . n}\right)\right) \quad=\operatorname{transform}(e) \cdot \mathrm{m}\left(e_{\star}^{\prime 1 . . n}\right) \\
& \text { where } e_{\star}^{\prime}=\operatorname{transform}\left(e_{\star}\right)^{1 . . n} \\
& \text { transform (throw) } \quad=\text { throw } \\
& \text { transform }(a ?\{\mathrm{x}: C \Rightarrow d\} /\{e\})=a^{\prime} ?\left\{\mathrm{x}: C \Rightarrow d^{\prime}\right\} /\left\{e^{\prime}\right\} \\
& \text { where } d^{\prime}=\text { transform }(d) \\
& \text { and } e^{\prime}=\operatorname{transform}(e) \\
& \operatorname{transform}\left(e_{\star}^{1 . . n} \operatorname{match}\left\{c_{\star}^{1 . . k}\right\}\right)=\operatorname{transform}\left(\text { rewrite }\left(e_{\star}^{1 . . n} \operatorname{match}\left\{c_{\star}^{1 . . k}\right\}\right)\right) \\
& \operatorname{transform}\left(\operatorname{def} \mathrm{m}\left(\mathrm{x}_{\star}: C_{\star}^{1 . . n}\right): C=\{e\}\right) \quad=\operatorname{def} \mathrm{m}\left(\mathrm{x}_{\star}: C_{\star}^{1 . . n}\right): C=\{\operatorname{transform}(e)\} \\
& \operatorname{transform}\left(\operatorname{class} C\left(\mathrm{x}_{\star}: C_{\star}^{1 . . n}\right) \triangleleft D\left\{a n_{\star} m d_{\star}^{1 \ldots k}\right\}\right)=\operatorname{class} C\left(\mathrm{x}_{\star}: C_{\star}^{1 . . n}\right) \triangleleft D\left\{a n_{\star} m d_{\star}^{\prime}{ }_{\star}^{1 . . k}\right\} \\
& \text { where } m d^{\prime}=\text { transform }(m d)
\end{aligned}
$$

